\title{
Distinct roles of KAP1, HP1 and G9a/GLP in silencing of the two-cell-specific retrotransposon MERVL in mouse ES cells
}

Irina A Maksakova ${ }^{1}$, Peter J Thompson ${ }^{1}$, Preeti Goyal' ${ }^{1}$, Steven JM Jones ${ }^{2}$, Prim B Singh ${ }^{3}$, Mohammad M Karimi ${ }^{1,2}$ and Matthew C Lorincz ${ }^{1 *}$

\begin{abstract}
Background: In mouse embryonic stem cells (mESCs), transcriptional silencing of numerous class I and II endogenous retroviruses (ERVs), including IAP, ETn and MMERVK10C, is dependent upon the H3K9 methyltransferase (KMTase) SETDB1/ ESET and its binding partner KAP1/TRIM28. In contrast, the H3K9 KMTases G9a and GLP and HP1 proteins are dispensable for this process. Intriguingly, MERVL retroelements are actively transcribed exclusively in the two-cell (2C) embryo, but the molecular basis of silencing of these class III ERVs at later developmental stages has not been systematically addressed.

Results: Here, we characterized the roles of these chromatin factors in MERVL silencing in mESCs. While MMERVK10C and IAP ERVS are bound by SETDB1 and KAP1 and are induced following their deletion, MERVL ERVs show relatively low levels of SETDB1 and KAP1 binding and are upregulated exclusively following KAP1 depletion, indicating that KAP1 influences MERVL expression independent of SETDB1. In contrast to class I and class II ERVs, MERVL and MERVL LTRdriven genic transcripts are also upregulated following depletion of G9a or GLP, and G9a binds directly to these ERVs. Consistent with a direct role for H3K9me2 in MERVL repression, these elements are highly enriched for G9a-dependent H3K9me2, and catalytically active G9a is required for silencing of MERVL LTR-driven transcripts. MERVL is also derepressed in HP1 $a$ and HP1 $\beta$ KO ESCs. However, like KAP1, HP1 $\alpha$ and HP1 $\beta$ are only modestly enriched at MERVL relative to IAP LTRs. Intriguingly, as recently shown for KAP1, RYBP, LSD1 and G9a-deficient mESCs, many genes normally expressed in the $2 \mathrm{C}$ embryo are also induced in HP1 KO mESCs, revealing that aberrant expression of a subset of $2 \mathrm{C}$-specific genes is a common feature in each of these $\mathrm{KO}$ lines.
\end{abstract}

Conclusions: Our results indicate that G9a and GLP, which are not required for silencing of class I and II ERVs, are recruited to MERVL elements and play a direct role in silencing of these class III ERVs, dependent upon G9a catalytic activity. In contrast, induction of MERVL expression in KAP1, HP1 a and HP1 $\beta$ KO ESCs may occur predominantly as a consequence of indirect effects, in association with activation of a subset of 2C-specific genes.

Keywords: G9a, GLP, HP1, KAP1, MERVL, ERV, Chimeric transcripts, Preimplantation, H3K9me2, mESC

\section{Background}

Endogenous retrovirus-like sequences (ERVs) are fossils of ancient retroviral integrations into the mammalian germline. Multiple independent colonization events have led to the accumulation of over 400 different ERV families with defined transcriptional patterns, often limited to specific developmental stages and cell types. Based on the

\footnotetext{
* Correspondence: mlorincz@mail.ubc.ca

'Department of Medical Genetics, Life Sciences Institute, 2350 Health Sciences Mall, University of British Columbia, Vancouver, British Columbia V6T 1Z3, Canada

Full list of author information is available at the end of the article
}

similarity of their reverse transcriptase genes, ERVs are grouped into three classes: I, II and III, most closely related to exogenous gammaretroviruses, betaretroviruses and spumaretroviruses, respectively [1]. Most ERVs in each class are no longer capable of transcription and/or retrotransposition due to the accumulation of mutations and/or efficient targeting by host silencing mechanisms that act at various stages of the viral life cycle [2]. Nevertheless, many ERVs possess functional regulatory sequences that direct transcription at specific developmental stages and/or in specific tissues. A number of ERV 
subfamilies are particularly active early in embryogenesis [3], likely due to selection for expression and in turn retrotransposition at those stages in development that maximize the likelihood of germline transmission.

The class III MT subfamily of MaLR retrotransposons, for example, which comprise less than $5 \%$ of the mouse genome [4], account for $13 \%$ of all transcripts in the fully grown oocyte [3]. The related class III ERV MERVL/ MuERV-L (mouse ERV with a leucine tRNA primerbinding site), of which there are 656 full-length copies and 37,172 solitary long terminal repeats (LTRs) in the C57BL/ 6 genome (based on Repeatmasker analysis), are among the first sequences to be transcribed in the early two-cell (2C) embryo and account for nearly $4 \%$ of the mouse transcriptome at the $2 \mathrm{C}$ stage [3,5-7]. Class II intracisternal A-type particle (IAP) ERVs on the other hand, of which there are well over 600 full-length copies in the mouse genome, only account for $0.6 \%$ of the $2 \mathrm{C}$ transcriptome [3]. Intriguingly, MERVL expression may be essential for development beyond the four-cell stage [5], perhaps due to the exaptation of ERV LTRs as promoters for essential genes [8,9]. Indeed, MERVL-driven genic transcripts are abundant at the $2 \mathrm{C}$ stage $[3,10]$ and in mouse embryonic stem cells (mESCs) that show 2C-like features [11,12]. Given that such transcripts are not detectable at later developmental stages, it is likely that the LTR promoters of such 'chimeric' genes are regulated by the same epigenetic mechanisms that govern the ERVs from which they are derived.

To minimize the generally deleterious effects associated with retrotransposition, a number of pathways have evolved to inhibit transcription of ERVs. DNA methylation, mediated by the de novo methyltransferases DNMT3A and DNMT3B and the maintenance methyltransferase DNMT1, plays a critical role in proviral silencing in somatic tissues [13-15] including fibroblasts $[16,17]$, as well as in late germline development $[18,19]$. Surprisingly however, while class I and II ERVs show broad DNA demethylation in G9a and GLP knockout (KO) mESCs [20], neither of these lysine methyltransferases (KMTases), which dimethylate lysine 9 of histone H3 (H3K9), are required for silencing of these ERVs. Furthermore, while IAP transcript levels are elevated in DNMT1-deficient relative to wild-type (wt) mESCs [21,22], this difference increases dramatically in $\mathrm{mESCs}$ cultured in the absence of leukemia inhibitory factor (LIF) [21], indicating that a DNA methylation-independent mechanism may also operate in undifferentiated ESCs to silence such ERVs.

Indeed, we recently reported that in mESCs, numerous class I and II ERVs, including MMERVK10C, MusD and ETn elements, are de-depressed in the absence of the H3K9 KMTase SETDB1/ESET, while IAP elements show the highest level of activation in the absence of both DNMT1 and SETDB1 [23,24]. Furthermore, robust silencing of each of these ERVs is dependent upon H3K9me3 deposited by SETDB1 $[24,25]$ and the corepressor KAP1/
TRIM28/TIF1- $\beta$, which directly interacts with the KAP1 interaction domain (KID) of SETDB1 [26]. As KAP1 can directly interact with any one of several hundred Krüppelassociated box zinc finger proteins (KRAB-ZFPs), we and others have proposed that the KAP1/SETDB1 complex may generally be recruited to ERVs by KRAB-ZFPs that recognize specific ERV sequences [23,24,27-30]. Curiously however, while class III MERVL elements are also upregulated in KAP1 KO mESCs [28], we observed only modest upregulation of these ERVs (approximately 2-fold) in SETDB1 KO mESCs [23].

Heterochromatin protein 1 (HP1) proteins, which encode chromo- and chromoshadow domains, function in both structural and gene regulatory pathways in eukaryotes [31-33]. These H3K9me 'readers' modulate gene expression in part through binding to $\mathrm{H} 3 \mathrm{~K} 9 \mathrm{me} 2 / 3$ via the chromodomain [34-39]. In addition, HP1 proteins directly interact with the PxVxL motif of KAP1 via their chromoshadow domain independent of H3K9 methylation state [29,40-42]. Intriguingly, this interaction is required for transcriptional silencing of reporter genes [34,43] as well as of the nonimprinted Mest allele in embryonal carcinoma cells [42]. Surprisingly however, we recently showed that depletion of HP1 $\alpha$ (encoded by $C b x 5$ ), HP1 $\beta$ (encoded by $C b x 1$ ) and/or HP1Y (encoded by $C b x 3$ ), alone or in combination, does not lead to derepression of class I or class II ERVs in mESCs [25], raising the question: do HP1 proteins play a role in repression of class III ERVs?

Here, using genetic knockouts and/or RNAi, we analyzed the roles of KAP1, HP1 $\alpha, H P 1 \beta$ as well as G9a and GLP, in transcriptional silencing of MERVL elements in mESCs. Our results indicate that MERVL expression is induced as a consequence of both direct and indirect effects, the former due to loss of H3K9me2 and the latter in association with derepression of genes normally expressed at the $2 \mathrm{C}$ stage.

\section{Results}

Class II ERVs are upregulated in KAP1- and SETDB1deficient cells, while MERVL ERVs are upregulated exclusively in KAP1-deficient cells

We recently showed that silencing of many class I and II ERV families is maintained in mESCs by SETDB1mediated deposition of H3K9me3 [23,24]. Consistent with a previous report [28], we also found that the SETDB1associated corepressor KAP1 is required for repression of many of the same ERVs [24], supporting an essential role for the KAP1-SETDB1 complex [44] in proviral repression. Curiously however, while MERVL elements were also reported to be dramatically upregulated in KAP1 KO mESCs [11,28], our genome-wide analysis revealed minimal derepression of this class III ERV family in SETDB1 KO mESCs [23]. Reanalysis of RNA-sequencing (RNA-seq) data from SETDB1 KO [23] and KAP1 KO mESCs [28], confirmed that many class I and II families are derepressed 
in both cell lines (Figure 1A-B). IAPEz and MMERVK10C (a close relative of IAP [45]) ERVs, for example, are upregulated 14- and 100-fold, respectively, in the SETDB1 $\mathrm{KO}$, versus 24- and 26-fold, respectively, in the KAP1 KO. In contrast, while MERVL elements, composed of three annotations in the UCSC genome browser: MT2_Mm (LTR), MERVL-int (internal region) and ORR1A3-int (Figure 1A), are upregulated 27-fold in the KAP1 KO, they are upregulated less than 3-fold in the SETDB1 KO (Figure 1B). Knockdown (KD) of Setdb1 or Kap1 by RNAi followed by qRT-PCR yielded similar results (Figure 1C), ruling out the possibility that the distinct phenotypes observed in SETDB1 versus KAP1 KO mESCs are due to differences in genetic background. Thus, while silencing of class III MERVL elements is indeed dependent upon KAP1, depletion of SETDB1 has a relatively modest effect on MERVL expression, revealing that KAP1 plays a role in silencing of this ERV subfamily independent of SETDB1.

To determine whether MERVL elements are bound by KAP1 and/or SETDB1, we performed meta-analysis of published KAP1 and SETDB1 chromatin immunoprecipitation sequencing (ChIP-seq) datasets [46,47]. Numerous class I and class II ERVs, including IAP subfamilies, show significant enrichment of both factors and a strong positive correlation between the two (Figure 1D), consistent with our previous observations that these ERV families are marked by H3K9me3 in a SETDB1-dependent manner [23]. In contrast, MERVL is one of a small group of ERVs showing no detectable enrichment of SETDB1 and low levels of cumulative KAP1 binding relative to most SETDB1-bound class I and II ERVs (Figure 1D). While class III MaLR ERVs ORR1A

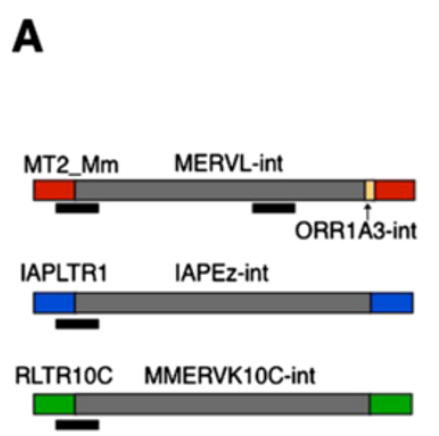

C

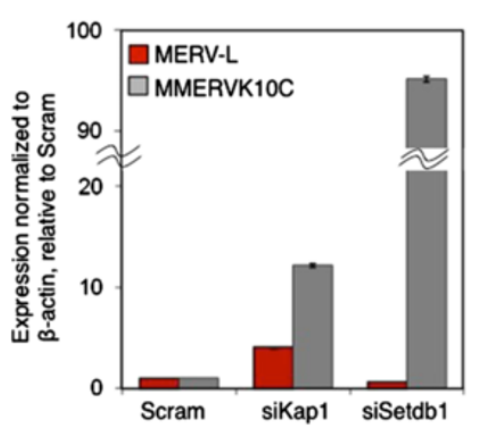

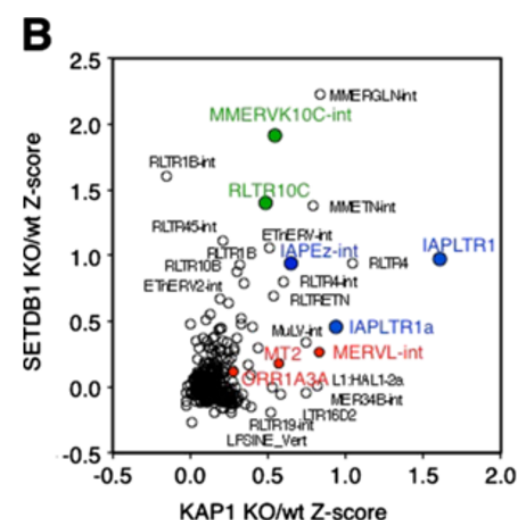

D

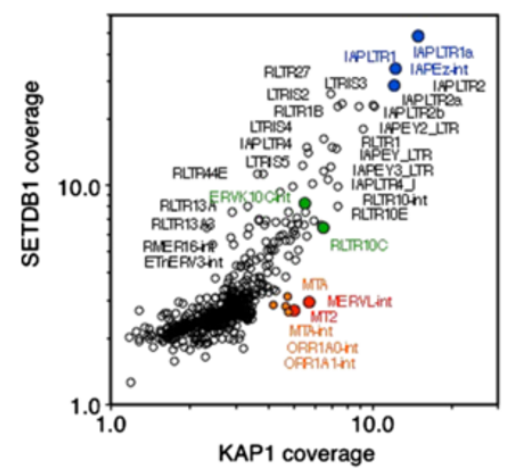

Figure 1 MERVL ERVs are derepressed upon KAP1 but not SETDB1 depletion, while MMERVK10C and IAP ERVs are upregulated following depletion of both. (A) Repbase annotations of the LTR and internal regions of full-length MERVL, IAPEZ and MMERVK10C elements are shown. Black bars indicate GPCR amplicons for LTRs of each family of element and the internal pol gene region for MERVL. (B) Deregulation of transposable element families in KAP1 and SETDB1 KO mESCs. RNA-seq data for SETDB1 [23] and KAP1 [28] KO lines and the corresponding wt cell lines were used to calculate Z-score values for all annotated retroelements and plotted as shown. (C) KAP1 and SETDB1 were depleted by RNAi in wt TT2 mESCs, and reactivation of MERVL and MMERVK10C elements was determined by qRT-PCR. Mean expression (+/-SD) of each ERV (normalized to $\beta$-actin) relative to a scrambled siRNA pool (Scram) is shown for three technical replicates. (D) MERVL is among a small group of transposable elements bound by KAP1 but not SETDB1. RPKM (*10) values generated from published ChIP-seq data for KAP1 [46] and SETDB1 [47] were plotted for all retroelements. Numerous class I and class II ERVs, including IAP subfamilies are enriched for both proteins, which are generally strongly correlated. MERVL elements are modestly enriched for KAP1, but show relatively low levels of SETDB1 coverage. ChIP-seq, chromatin immunoprecipitation sequencing; RPKM, reads per kilobase per million mapped reads. 
and MTA are enriched for KAP1 at levels similar to MERVL, only MERVL is upregulated in KAP1 KO mESCs. To determine whether KAP1 binding at MERVL elements is associated with enrichment of KAP1 in the unique regions flanking these proviruses, we analyzed KAP1 enrichment in the nonrepetitive sequences flanking all 656 full-length MERVL, 298 MMERVK10C and 599 IAPEz elements. As shown previously for H3K9me3 [25,48], KAP1 binding is clearly higher in the immediate flanks of IAP elements when analyzed in aggregate, decreasing to background levels with increasing distance to the provirus (Figure 2A). MMERVK10C
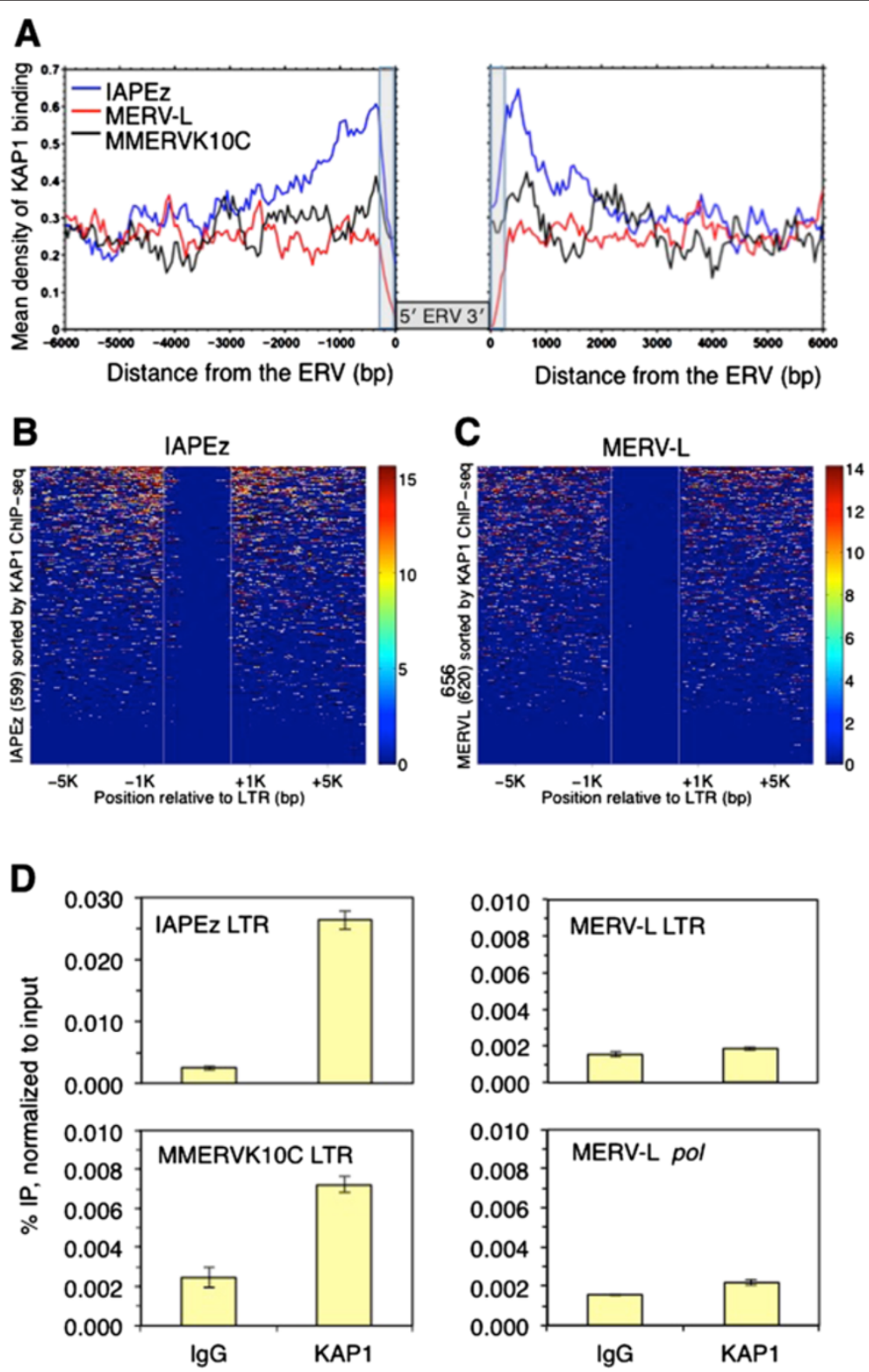

Figure 2 Unique regions flanking IAPEz but not MERVL elements are highly enriched in KAP1. (A) Profiling of KAP1 in the flanking sequence of all full-length IAPEz, MERVL and MMERVK10C ERVs. KAP1 ChIP-seq reads [46] from wt mESCs were aligned to the mouse genome (mm9), and the density profile of unique reads mapping to the $6 \mathrm{~kb}$ regions flanking all annotated intact MERVL (656), MMERVK10C (298) and IAPEz (599) elements, was plotted as shown. (B-C) Heat maps of KAP1 enrichment in the genomic regions flanking 599 IAPEz and 656 MERVL elements in wt mESCs. KAP1 ChIP-seq reads [46] were aligned to the mouse genome (mm9), and the density of uniquely aligned reads, mapping to the $6 \mathrm{~kb}$ regions flanking all intact ERVs of the specified families, was plotted. Reads extending into the ERV are due to in silico extension of aligned reads by 300 bp. (D) ChIP and qPCR analysis of KAP1 in TT2 wt mESCs at the LTRs of IAPEz, MMERVK10C and MERVL, as well as the MERVL pol internal region. IgG, negative control IP. Data are mean enrichment from three technical replicates as a percentage of the input chromatin and error bars represent SD. IgG, immunoglobulin G; IP, immunoprecipitation; SD, standard deviation. 
elements also show enrichment in proximal versus distal flanking regions, although to a lesser extent. This pattern is common to multiple individual IAP elements (Figure 2B); consistent with the hypothesis that KAP1 binds directly to this class II ERV and spreads into neighboring genomic regions. In contrast, enrichment of KAP1 is not detected at the flanks of MERVL elements when analyzed in aggregate (Figure 2A) and is sparsely detected at the flanks of only a small fraction of MERVL elements when analyzed individually (Figure 2C). Furthermore, in contrast to the sequences flanking individual IAP elements, enrichment is evenly distributed across the $6 \mathrm{~kb}$ regions flanking the few individual MERVL elements that show KAP1 binding in their flanks, indicating that the low level of KAP1 enrichment observed within these elements may reflect the chromatin state of the locus/integration site, rather than direct recruitment of KAP1 to these elements. Alternatively, in the absence of SETDB1-mediated H3K9me3 deposition, spreading of KAP1 into the regions flanking MERVL elements may not occur, leading to a relatively low level of focal KAP1 enrichment within the regulatory region of these ERVs. As an alternative approach to quantify KAP1 enrichment specifically within MERVL elements, we conducted ChIP using a KAP1-specific antibody. Consistent with the ChIP-seq data, KAP1 enrichment was detected at IAP and to a lesser extent at MMERVK10C LTRs (Figure 2D). In contrast, no enrichment was detected at LTR or pol internal regions of MERVL, using primers that detect 519 and 637 elements, respectively, as determined by in silico PCR. While we cannot rule out the possibility that relatively weak, localized binding of KAP1 to MERVL LTRs plays a direct, SETDB1independent role in silencing of these elements, SETDB1/H3K9me3 is routinely observed at repressed native loci and transgenes bound by KAP1 [23,26,29]. Furthermore, KAP1 mutants that cannot interact with SETDB1 are defective in silencing of KAP1-bound transgenes [26]. Thus, our observations are also consistent with the hypothesis that derepression of MERVL elements in KAP1-deficient mESCs occurs as a consequence of indirect effects.

\section{MERVL elements and chimeric transcripts are upregulated in G9a and GLP KO mESCs}

While we have shown previously that silencing of class I and II ERVs is not dependent upon G9a [20], we did not address whether this $\mathrm{H} 3 \mathrm{~K} 9 \mathrm{me} 1 / 2 \mathrm{KMTase}$ plays a role in repression of class III ERVs. As MERVL elements were recently shown to be marked by H3K9me2 [11], we next tested whether G9a and the closely related KMTase GLP,
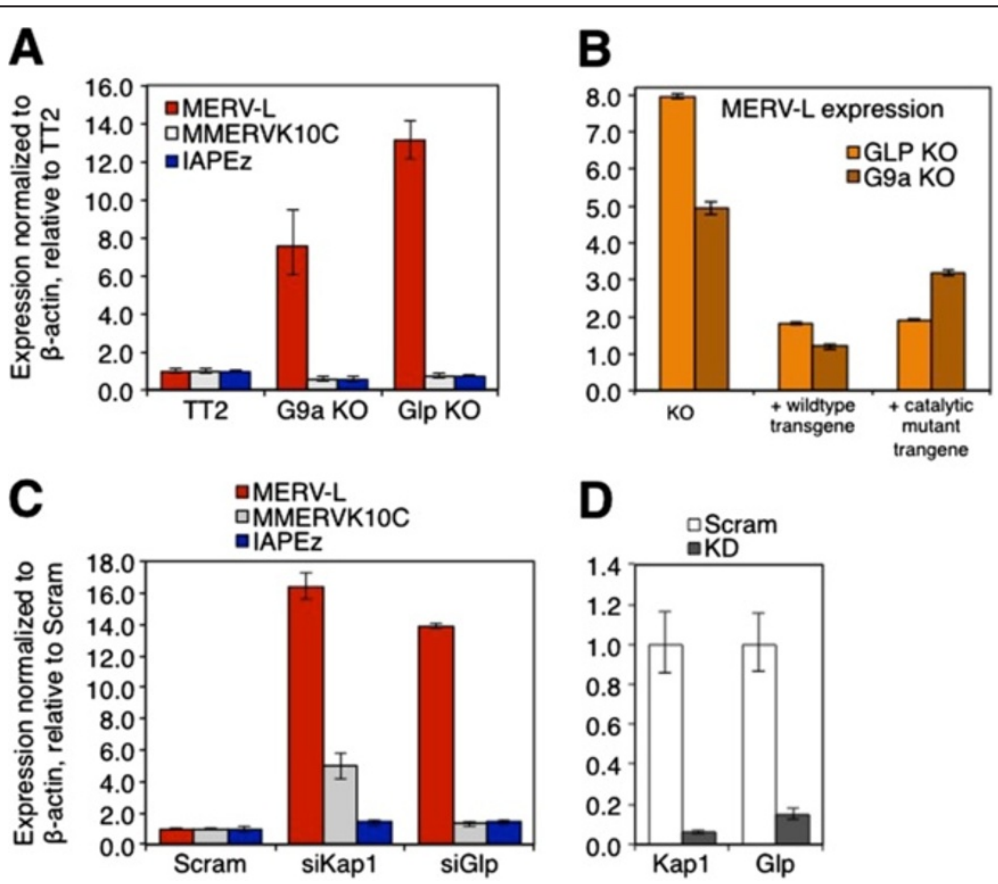

Figure 3 MERVL ERVs are derepressed in G9a- and GLP-deficient mESCs and MERVL silencing is dependent on G9a catalytic activity. (A) Upregulation of MERVL in G9a and GLP KO mESCs. Expression of MERVL, MMERVK10C and IAPEz ERVs in TT2 wt, G9a and GLP KO mESCs was analyzed by qRT-PCR. Mean (+/-SD) expression levels relative to the wt line for three technical replicates (normalized to $\beta$-actin) are shown. (B) Catalytic activity of G9a but not GLP is required for MERVL silencing. G9a or GLP KO mESCs stably expressing wt or catalytic mutant G9a (C1168A) or GLP (C1201A) [50] transgenes, respectively, were assessed for MERVL expression by qRT-PCR, as described above. (C) MERVL but not MMERVK10C or IAPEz ERVs are upregulated upon KD of G/p. Relative expression of ERVs was determined by qRT-PCR, as described above. (D) Efficiency of each KD was determined by qRT-PCR with primers specific for Kap1 and Glp, as described above. SD, standard deviation. 
which form a heterodimer [49], are required for silencing of MERVL ERVs. Quantitative RT-PCR analysis of G9a KO and GLP KO mESCs revealed that MERVL elements are upregulated approximately 8-fold and approximately 13-fold respectively, relative to their parent line TT2 (Figure 3A). In contrast, MMERVK10C, similar to IAP ERVs (Figure 3A and [20]), showed no change in expression in either the G9a or GLP KO lines. Analysis of G9a or GLP KO mESCs stably expressing wt or catalytic mutants of G9a (G4, C1168A) or GLP (L4, C1201A), respectively [50], revealed that MERVL silencing is dependent only upon catalytically active G9a (Figure 3B). This result may be explained by the fact that while G4 and L4 catalytic mutants form heteromeric complexes with wt GLP and G9a, respectively, H3K9me2 levels are restored only in the GLP KO line rescued with the L4 catalytic mutant [50], implicating G9a as the critical H3K9 KMTase in the context of the G9a/GLP heterodimer. Transient depletion of GLP also disrupts MERVL silencing (siRNAs directed against G9a did not yield efficient depletion of $G 9 a$ mRNA), with a 14-fold increase in MERVL expression observed 4 days post siRNA transfection (Figure 3C-D). In contrast, as expected, no increase in MMERVK10C or IAPEz expression was observed following KD of Glp. While MERVL expression was induced approximately 16-fold following KAP1 KD, MMERVK10C was induced only approximately 5-fold in this experiment and IAPEz - only 1.5-fold, likely due to DNA methylationmediated repression [23] and/or insufficient depletion of the protein. Importantly, KAP1 and LSD1 protein levels are not reduced in GLP or G9a KO mESCs (Additional file 1: Figure S1A), indicating that derepression of MERVL elements in these cells is not due to destabilization of these proteins, which were previously implicated in MERVL silencing $[11,28]$. Furthermore, while interactions between KAP1 and HP1 $\beta$ and G9a and HP1 $\beta$ were clearly detected by co-immunoprecipitation (co-IP), as reported previously $[40,41,51]$, no interaction between KAP1 and G9a was detected under the same conditions (Additional file 1: Figure S1B), indicating that G9a is unlikely to regulate MERVL elements via direct interaction with KAP1.

LTRs that are derepressed in mESCs deficient in SETDB1 or the H3K4me1/2 demethylase LSD1/KDM1A can function as alternative promoters for downstream genes [11,23], and the specific ERV families upregulated in these KOs contribute to the majority of such aberrantly expressed chimeric transcripts. To determine whether naturally occurring chimeric transcripts that initiate in MERVL/MT2 LTRs and splice to downstream genic exons are also upregulated in G9a- or GLP-depleted mESCs, we first identified all protein-coding genes represented in both RefSeq and ENSEMBL databases with an MT2 LTR as the annotated exon 1 (Additional file 2). Among the 43 genes on this list, 10 and 6 were upregulated $>4$-fold in KAP1 and G9a $\mathrm{KO}$ mESCs, respectively, relative to their wt parent lines, as determined by meta-analysis of previously published RNAseq data $[12,28]$. Strikingly, of the six upregulated genes in the G9a KO line, five are also upregulated in the KAP1 KO line. The MT2B LTR-driven gene Zfp352, which is upregulated dramatically in both of these $\mathrm{KO}$ lines, is induced approximately 80-fold in Glp KD mESCs, as determined by qRT-PCR analysis (Additional file 1: Figure S1C), confirming that at least a subset of MERVL LTR-driven genic transcripts are silenced by G9a and GLP. Together, these data demonstrate that the G9a/GLP complex is not only required for silencing of intact MERVL elements but also plays a critical role in silencing of a subset of the annotated genes that initiate in MERVL LTRs.

\section{MERVL elements are direct targets of the G9a/GLP H3K9 KMTase complex}

To determine whether G9a is directly bound at MERVL elements, we performed cross-linked ChIP in wt and G9a KO mESCs using a G9a-specific antibody. In contrast to KAP1, G9a was specifically enriched in the LTR and pol gene regions of MERVL (Figure 4A) and was also detected at IAPEz and MMERVK10C LTRs (Figure 4B). Importantly, G9a was depleted at these regions in the G9a KO line, confirming the specificity of this antibody. To determine whether MERVL elements are marked by H3K9me2 in a G9a-dependent manner, we analyzed all three states of H3K9 methylation in wt and G9a KO mESCs by native ChIP (N-ChIP). H3K9me2 is highly enriched at the MERVL 5'LTR/promoter region in wt $\mathrm{mESCs}$ and reduced to near background levels in G9a KO mESCs (Figure 4C). In contrast, the $5^{\prime} \mathrm{LTR} /$ promoter regions of MMERVK10C and IAPEz ERVs show relatively low but clearly detectable levels of H3K9me2 in wt mESCs (Figure 4D-E), as shown previously [20]. The converse is true for H3K9me3, consistent with our previous observations that class II ERVs, including IAPEz and MMERVK10C, are directly regulated by SETDB1 $[23,24]$ and the results presented above, which reveal that MERVL elements are directly regulated by G9a and GLP. Taken together, these results indicate that the G9a/GLP heterodimer plays a direct role in silencing of MERVL elements.

\section{MERVL and MERVL-promoted chimeric genes are upregulated in HP1 $\alpha$ and HP1 $\beta$ KO mESCs}

HP1 proteins are thought to play an important role in transcriptional silencing via binding to methylated H3K9, leading to chromatin compaction and heterochromatin spreading [29,35,37]. While these corepressors have highest affinity for H3K9me3, they can also bind H3K9me2 in vitro and have been reported to directly interact with both KAP1 and the G9a/GLP complex [35,40,41,51]. To determine whether HP1 proteins are required for silencing of MERVL elements, we generated RNA-seq data for mESCs deficient in HP1 $\alpha$ or HP1 $\beta$ and the wt parent line HM1 (described in [25]). 
A

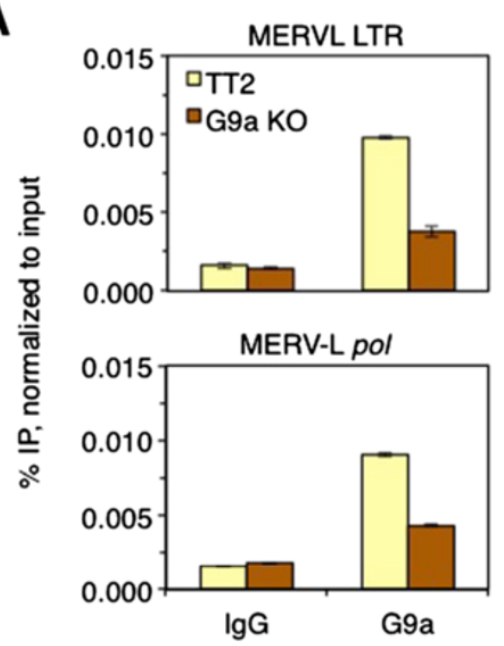

B

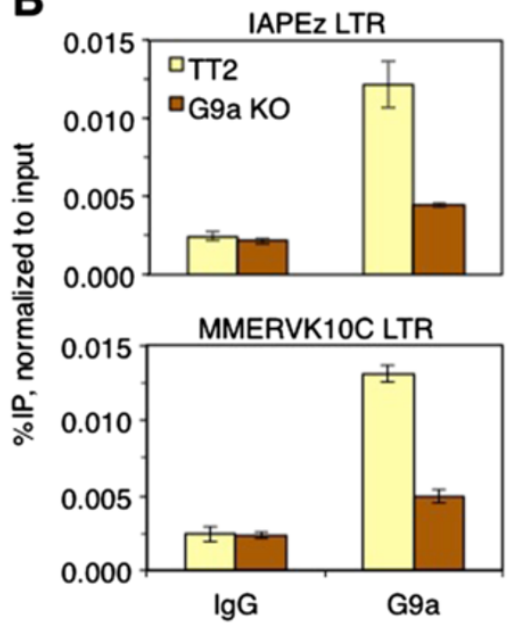

E
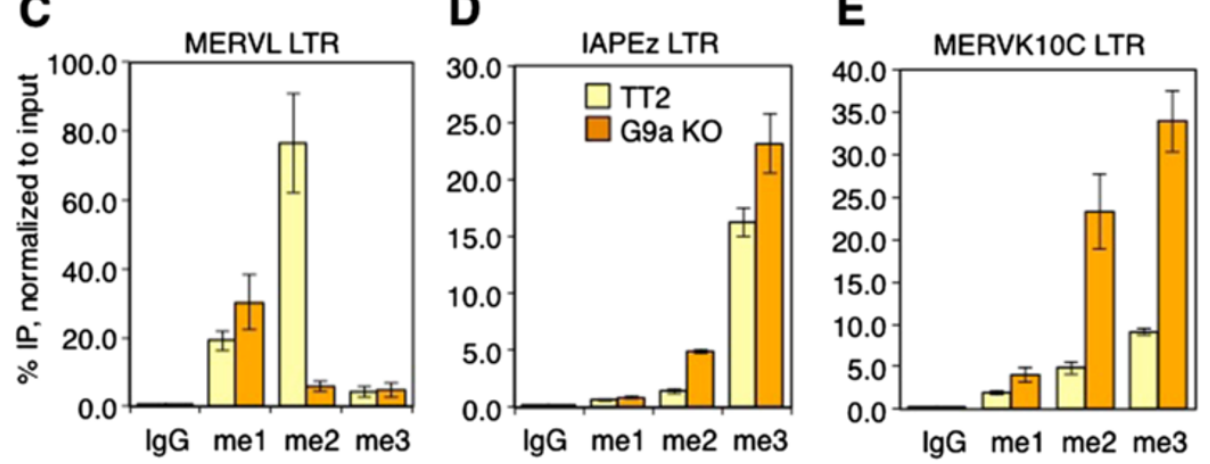

Figure 4 G9a is bound at MERVL and H3K9me2 enrichment at MERVL elements is dependent on G9a. (A-B) ChIP and qPCR of G9a in TT2 wt and G9a KO mESCs at LTR of MERVL and MERVL internal region and LTRs of IAPEz and MMERVK10C. IgG, negative control IP. Data are mean enrichment from three technical replicates as a percentage of the input chromatin and error bars represent SD. (C-E) N-ChIP and qPCR was performed for H3K9me1 (me1), H3K9me2 (me2), H3K9me3 (me3) and IgG as a negative control in TT2 wt and G9a KO mESCs at the LTRs of MERVL, IAPEz and MMERVK10C. Data are mean enrichment (+/-SD) for three technical replicates normalized to input. IgG, immunoglobulin G; IP, immunoprecipitation; SD, standard deviation.

Intriguingly, MERVL elements are among the most highly upregulated retrotransposons in both $\mathrm{HP} 1 \alpha$ and $\mathrm{HP} 1 \beta$ $\mathrm{KO}$ lines (Figure 5A), showing increases in expression of 6-fold and 11-fold, respectively, relative to the parent HM1 mESC line. These elements are also among the few ERVs upregulated in both HP1 and KAP1 KO mESCs (Additional file 1: Figure S2A). Consistent with the RNAseq data, qRT-PCR analysis revealed that MERVL elements are upregulated approximately 4-fold and approximately 6-fold in HP1 $\alpha$ and HP1 $\beta$ KO mESCs, respectively (Figure 5B). Importantly, analysis of RNA-seq reads that uniquely align to specific full-length MERVL elements, of which there are 656 genomic copies, reveals that the same elements are de-repressed in the HP1 $\alpha \mathrm{KO}$ and $\mathrm{HP} 1 \beta \mathrm{KO}$ lines (Additional file 1: Figure S2B).

To determine whether genes that initiate in MERVL LTRs are also derepressed in HP1-depleted ESCs, we calculated the expression levels of each of the 43 annotated MT2-initiated genic transcripts. Four and six of these genes were upregulated $>4$-fold in the HP1 $\alpha$ and $\mathrm{HP} 1 \beta \mathrm{KO}$ lines, respectively, relative to the wt parent line HM1 (Additional file 2). Strikingly, four of the genes in each case were also among the genes upregulated in the KAP1 and G9a KO lines. Zfp352 for example, showed an increase in expression of 14- and 28 -fold in the HP1 $\alpha$ and HP1 $\beta$ KO lines, respectively. To determine whether additional unannotated genic transcripts (that is transcripts not present in the RefSeq or ENSEMBL databases) initiate in MERVL/MT2 LTRs in HP1 mutant cells, we identified all transcripts in our paired-end RNA-seq data in which one of the mate pairs aligns to an LTR element and the other to an annotated genic exon and scored all genes having $>5$ unique support reads for each in HM1 (wt), HP1 $\alpha$ and HP1 $\beta$ KO mESCs. While ERVs of all three classes are represented among constitutively expressed chimeric transcripts in wt mESCs (Additional file 1: Figure S2C), the number of MERVL LTR-driven chimeric genes is 

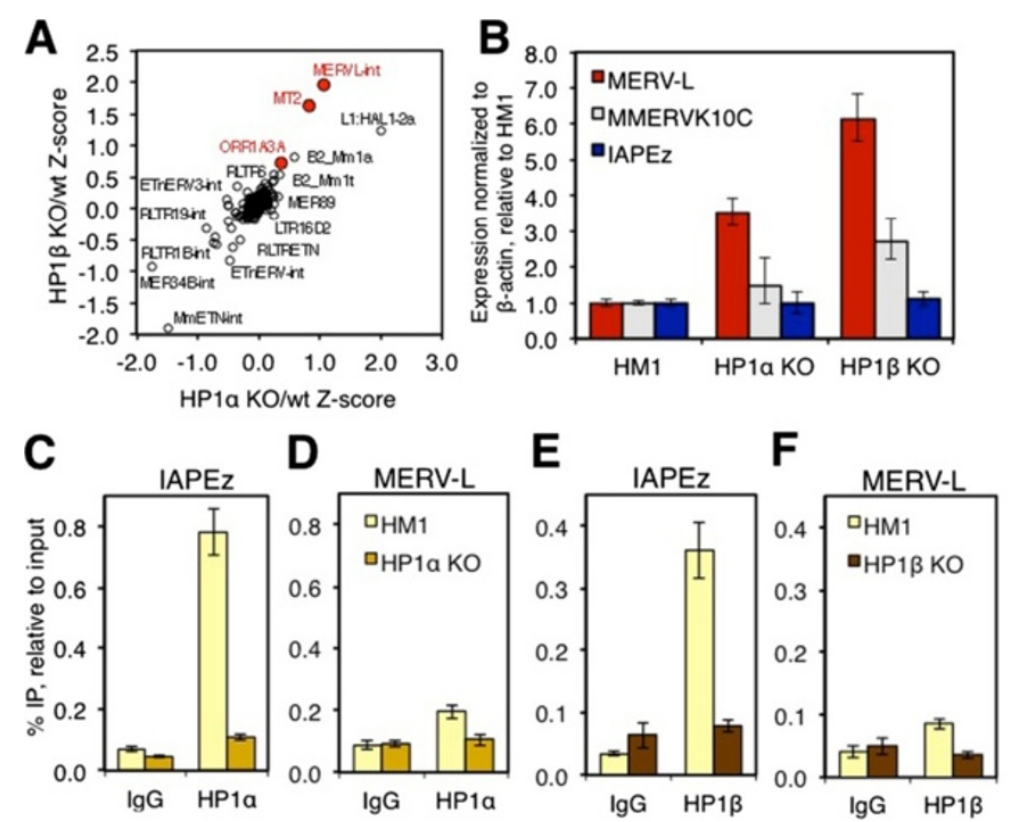

Figure 5 MERVL ERVs are derepressed in HP1 $\alpha$ and HP1 $\beta$ KO mESCs but HP1 $\alpha$ and HP1 $\beta$ show relatively low enrichment at these ERVs. (A) RNA-seq analysis of retroelement expression in HP1a and HP1 $\beta$ KO mESCs. RNA-seq data for HP1a and HP1 K KO mESCs and the HM1 parent line was generated, and Z-scores were calculated for all retroelements and plotted. Note that MERVL elements show relatively high levels of derepression in both KO lines. (B) MERVL elements are upregulated in HP1a and HP1 $\beta$ KO mESCs. Expression of MERVL, IAP and MMERVK10C ERVs was analyzed by qRT-PCR in HM1 wt, HP1a and HP1 $\beta$ KO lines. Mean (+/-SD) expression levels (normalized to $\beta$-actin) are shown, relative to the wt line for three technical replicates. (C-F) IAPEz elements show significantly higher levels of HP1a and HP1 $\beta$ enrichment than do MERVL elements. Cross-linked ChIP was performed with antibodies specific for HP1a or HP1 $\beta$ in HM1 and the corresponding KO cell line. IgG was used as a negative control. The level of enrichment for each IP was determined by QPCR, and the mean and standard deviation of three technical replicates are shown for each experiment. IgG, immunoglobulin G; IP, immunoprecipitation; SD, standard deviation.

higher in HP1 KO mESCs, with MT2 transcripts contributing $19 \%$ and $28 \%$ of all chimeric transcripts in the HP1 $\alpha$ (15 of 78 total) and HP1 KO (28 of 102 total) lines, respectively, compared to $12 \%$ (5 out of 43 ) in wt cells (Additional file 1: Figure S2C). Together, these data demonstrate that $\mathrm{HP} 1 \alpha$ and $\mathrm{HP} 1 \beta$ are required for silencing of MERVL elements as well as genic transcripts that initiate in MERVL LTRs.

\section{HP1 $\alpha$ and HP1 $\beta$ are only modestly enriched at the 5'LTR of MERVL}

To determine whether HP1 proteins are enriched specifically at MERVL LTRs, HP $1 \alpha$ and HP1 $\beta$ binding was analyzed via ChIP-qPCR using chromatin extracts isolated from HM1 wt and HP1 KO mESCs. An approximately 8 -fold higher level of enrichment of HP1 $\alpha$ was detected in the 5 'LTR region of IAPEz elements relative to the immunoglobulin G (IgG) control immunoprecipitation (IP), consistent with our previous observations [24] (Figure 5C). This enrichment was lost in HP1 $\alpha \mathrm{KO}$ mESCs, confirming the specificity of the HP1 $\alpha$ antibody. Importantly, HP1 $\alpha$ binding was also reduced at IAPEz elements and at the promoter region of the single-copy imprinted gene Mest following KD of Kap1 (Additional file 1: Figure S2D-E), confirming that $\mathrm{HP} 1 \alpha$ binding to these loci is KAP1dependent [42]. In contrast, the level of HP1 $\alpha$ enrichment observed in the 5'LTR region of MERVL elements was only approximately 2 -fold higher than the IgG control IP (Figure 5D), despite comparable numbers of genomic copies recognized by the MERVL LTR (519 elements) and IAP LTR (638 elements) primer pairs, which were also used in the analysis of H3K9 methylation state described above. A similar pattern was observed for $\mathrm{HP} 1 \beta$, which is enriched approximately 7-fold and 2-fold at IAPEz and MERVL LTRs, respectively (Figure 5E-F). Thus, while MERVL elements are upregulated in HP1 $\alpha$ and HP1 $\beta$ KO mESCs, these KAP1-interacting factors, like KAP1 itself, are enriched at relatively low levels at MERVL LTRs.

\section{C-specific genes are induced in KAP1, G9a, HP1a and HP1 $\beta$, KO mESCs}

Intriguingly, an increase in the percentage of cells permissive for MERVL expression and upregulation of numerous transcripts normally expressed at the $2 \mathrm{C}$ stage was recently reported for KAP1-, LSD1- and G9a-deficient mESCs [12]. These observations raise the possibility that MERVL 
elements are derepressed in these $\mathrm{KO}$ lines at least in part as a result of indirect effects of establishment of a cellular fate permissive for MERVL transcription. To determine whether $2 \mathrm{C}$-specific genes are also upregulated in $\mathrm{HP} 1 \alpha$ and/or HP1 $\beta$ KO mESCs, we first generated a list of genes expressed specifically at this embryonic stage, based on published RNA-seq data from single blastomeres [52]. We identified genes that are expressed at levels at least 4-fold higher at the $2 \mathrm{C}$ stage than the oocyte or eight-cell stages (oocyte $<2 \mathrm{C}>8 \mathrm{C}$ ). Of 264 such $2 \mathrm{C}$-'specific' genes, $11 \%$ and $6 \%$ were upregulated relative to their wt parent lines in KAP1 and G9a KO mESCs, respectively (Figure 6A). Strikingly, the transcription start sites (TSSs) of nine of the sixteen $2 \mathrm{C}$-specific genes upregulated in the $\mathrm{G9a} \mathrm{KO}$ line overlap with or are within $5 \mathrm{~kb}$ of a MERVL/MT2 LTR. Similarly, analysis of the HP1 $\alpha$ and HP1 $\beta$ KO RNA-seq datasets presented above revealed that $7 \%$ and $15 \%$ of $2 \mathrm{C}$ specific genes are upregulated in these lines respectively, relative to the parent HM1 line. Taken together, 16\% (42/ 264) of $2 \mathrm{C}$ genes are upregulated in one or both of the HP1 KO lines (Figure 6B). 2C-specific genes are significantly overrepresented among the genes upregulated in these $\mathrm{KO} \mathrm{mESCs}$, as only $1 \%$ of all genes in the blastomere RNA-seq data (of which there are 26,155) were identified as $2 \mathrm{C}$-specific. Furthermore, of the nine $2 \mathrm{C}$-specific genes upregulated in all four $\mathrm{KO}$ lines (Figure 6C), seven initiate transcription either within an MT2 LTR or within $5 \mathrm{~kb}$ of an MT2, MT2A or MT2C LTR, whereas only 4.5\% (1387/ 30321) of all RefSeq genes are within $5 \mathrm{~kb}$ of an MT2, MT2A or MT2C annotated LTR.

Consistent with the observation that a subset of $2 \mathrm{C}$ genes are upregulated in these $\mathrm{KO}$ lines, genes shown previously to be expressed exclusively in early embryogenesis (confirmed to be $2 \mathrm{C}$-specific in the analysis described above), including Zfp352 [53], Zscan4d [54] and Tdpoz3-4 [55] (Figure 6D), are upregulated in $\mathrm{HP} 1 \alpha$ and $\mathrm{HP} 1 \beta \mathrm{KO}$ mESCs as well as KAP1 and G9a KO mESCs (Figure 6E). Moreover, the top five genes showing the greatest difference in expression between the $2 \mathrm{C}$ stage and the oocyte and 8C stages: Zfp352, Gm5039, Gm8994, B020004J07Rik and Dub1a, also showed dramatic upregulation in HP1 $\alpha$ and HP1 $\beta$ KO mESCs (Additional file 1: Figure S3A-B). Taken together, these observations reveal that, like KAP1 and G9a KO ESCs, HP1 KO mESCs show a significant increase in expression of a specific subset of $2 \mathrm{C}$-specific genes, many of which are regulated by MERVL LTR promoters.

\section{Discussion}

MERVL elements are present in all placental mammals, suggesting that a common mammalian ancestor was colonized at least 70 million years ago. Several bursts of amplification have subsequently occurred in a number of lineages, including the mouse [56], which now harbors 600 to 700 full-length copies and approximately 37,000 solitary LTRs in the C57BL/6 genome. Intriguingly, a subset of MERVL LTRs may have been domesticated to serve as gene promoters specifically at the two-cell stage, when MERVL LTR promoters are highly transcribed $[3,10]$. While a small subset of sequences derived from ERVs may play a positive role by providing regulatory signals or encoding exapted proteins, proviral integration events are more likely to compromise host fitness. To counteract this threat, multiple mechanisms directed at various stages of the viral life cycle have evolved, including at the transcriptional level [57-60]. Our results reveal that distinct H3K9 methylation-based mechanisms of transcriptional silencing are used against different ERV families. At numerous class I and II ERVs, SETDB1 is recruited by KAP1 $[44,61]$, which in turn interacts with one of multiple KRAB-zinc finger proteins that presumably recognize specific sequences within these ERVs to promote $\mathrm{H} 3 \mathrm{~K} 9$ me3-mediated transcriptional silencing [44,61] (Figure 7A). Indeed, these parasitic elements are dramatically upregulated in KAP1- and SETDB1-deficient mESCs $[24,28]$. The relatively high levels of H3K9me3 [23,24], KAP1 and HP1 detected in the LTR and flanking genomic regions of class I and II ERVs, particularly IAP elements, is most consistent with a 'spreading' model, whereby deposition of H3K9me3 induces binding of HP1 proteins $[35,36]$ and in turn KAP1 and SETDB1, which promotes deposition of H3K9me3 at neighboring nucleosomes in a process that occurs reiteratively. Curiously however, class I and II ERVs are not derepressed in MESCs deficient in HP1 proteins [25], leaving the role of HP1 proteins at class I and II ERVs in question.

Unlike class I and II ERVs, MERVL elements are neither bound by SETDB1 nor marked by H3K9me3, and deletion of SETDB1 does not dramatically induce expression of these class III ERVs. Furthermore, while deletion of KAP1, HP1 $\alpha$ and HP1 $\beta$ promotes upregulation of MERVL elements, we did not detect significant enrichment of these factors at MERVL elements. While we cannot rule out the possibility that our ChIP assay is not sufficiently sensitive to detect binding of these chromatin factors to MERVL elements, we did detect significant enrichment of KAP1, HP1 $\alpha$ and HP1 $\beta$ at IAP elements in the same experiments. A compelling explanation for the apparently distinct mechanisms underlying repression of these ERVs may be that MERVL elements are not bound by any of the over 300 KRAB-ZFPs encoded in the mouse genome $[62,63]$. While the striking diversity in KRAB-ZFPs was likely driven by selection for KRAB-ZFPs that recognize specific motifs within ERVs [64], MERVL-specific KRAB-ZFPs may simply not have evolved since the two bursts of MERVL retrotransposition in the mouse genome, which occurred 2 and 4 million years ago [56].

While this manuscript was under preparation, Macfarlan et al. reported that MERVL elements are upregulated in 


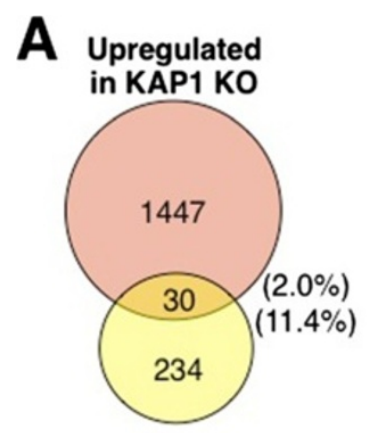

Upregulated in G9a KO

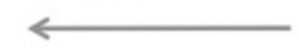

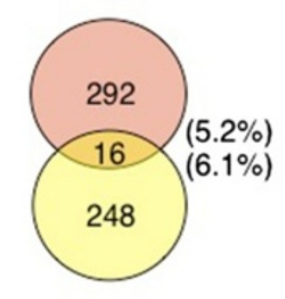

$2 \mathrm{C}$ specific $(\mathrm{O} 0<2 \mathrm{C}>8 \mathrm{C})$ genes
B Upregulated in:
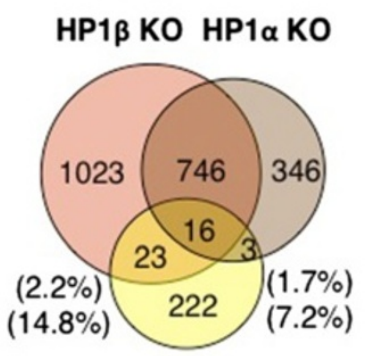

$2 \mathrm{Cspecilic}(\mathrm{O}<<2 \mathrm{C}>\mathrm{BC})$ genes

$\begin{array}{cll}\text { 2C genes upregulated in KAP1, G9a, HP1 } \alpha \text { AND HP1 } \beta \text { KO mESCS } \\ \text { BefSeqD } & \text { Gene_D } & \text { MERVLLTR distance to the TSS } \\ \text { NM_001007579 } & \text { BC080695 } & \text { MT2 } ~ 5 \mathrm{~kb} \text { upstream } \\ \text { NM_001033790 } & \text { B020004J07Rik } & \text { MT2 promoter } \\ \text { NM_001034896 } & \text { Tmem92 } & \text { MT2A 7kb upstream } \\ \text { NM_007831 } & \text { Dcc } & \text { MT2 near 3' end of gene chimaeric with 3' genic exons } \\ \text { NM_009705 } & \text { Arg2 } & \text { MT2 2kb upstream } \\ \text { NM_153102 } & \text { Zfp352 } & \text { MT2 promoter } \\ \text { NM_201409 } & \text { Dub1a } & \text { MT2C 2 kb upstream } \\ \text { NR_003647 } & \text { Gm5039 } & - \\ \text { NM_001085412 } & \text { Gm13078 } & \text { MT2 } ~ 5 \mathrm{~kb} \text { upstream }\end{array}$
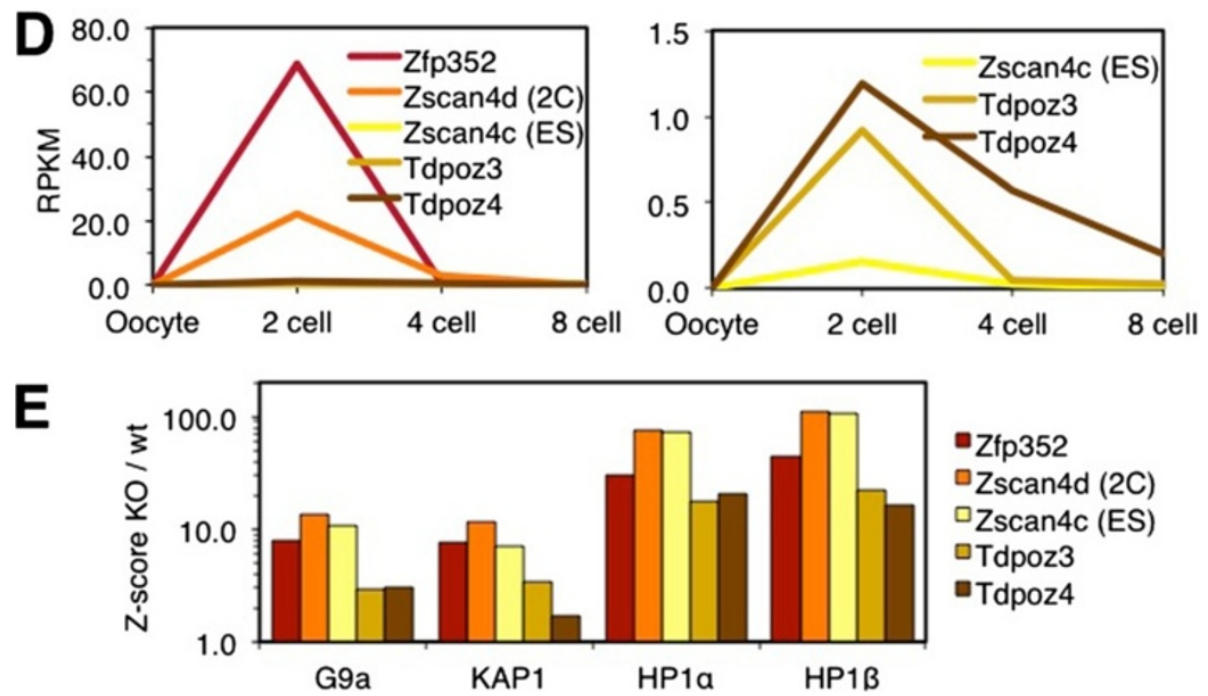

-Zfp352

-Zscan4d (2C)

Zzscan4c (ES)

-Tdpoz3

-Tdpoz4

Figure 6 Two-cell embryo-specific genes are induced in HP1a, HP1 $\beta$, KAP1 and G9a KO mESCs. A list of two-cell (2C) specific genes was produced from single blastomere expression data [52] by identifying genes expressed at levels 4 -fold higher at the $2 \mathrm{C}$ stage than the oocyte or $8 \mathrm{C}$ stages $(\mathrm{O} \circ<2 \mathrm{C}>8 \mathrm{C})$. (A-B) Venn diagrams illustrating the overlap between this gene list and the list of genes upregulated at least 4 -fold in KAP1, G9a [12,28], HP1a or HP1 $\beta$ KO mESCs are shown. The percentage of genes upregulated in the KO that are also 2C-specific is displayed above, while the percentage of $2 \mathrm{C}$-specific genes that are also upregulated in the $\mathrm{KO}$ are presented below. (C) The nine genes upregulated in all four KO lines are listed, along with the distance of the nearest MERVL LTR (MT2) to the transcription start site (TSS). (D) Confirmation of the expression pattern of previously identified 2C-specific genes. RPKM values, derived by division of reads per million (RPM) values from RNA-seq data generated from pooled single blastomeres [52] by transcript length, are presented for Zfp352, Zscan4d, Zscan4c, Tdpoz3 and Tdpoz4 genes. (E) Expression levels of these 2C-specific genes was determined in G9a, KAP1, HP1a and HP1 $\beta$ KO mESCs as well as their wt parent lines using our RNA-seq data (HP1a and HP1 $\beta$ ) or previously published RNA-seq data (G9a and KAP1 [12,28]), and Z-score values (see Materials and Methods) for each are presented.

G9a KO mESCs [12], although the molecular basis of aberrant MERVL expression was not addressed. Here, we confirm this observation, and extend it to include mESCs deficient in the related KMTase GLP. Furthermore, we show that MERVL elements are bound by G9a and that maintenance of MERVL repression is dependent upon the 


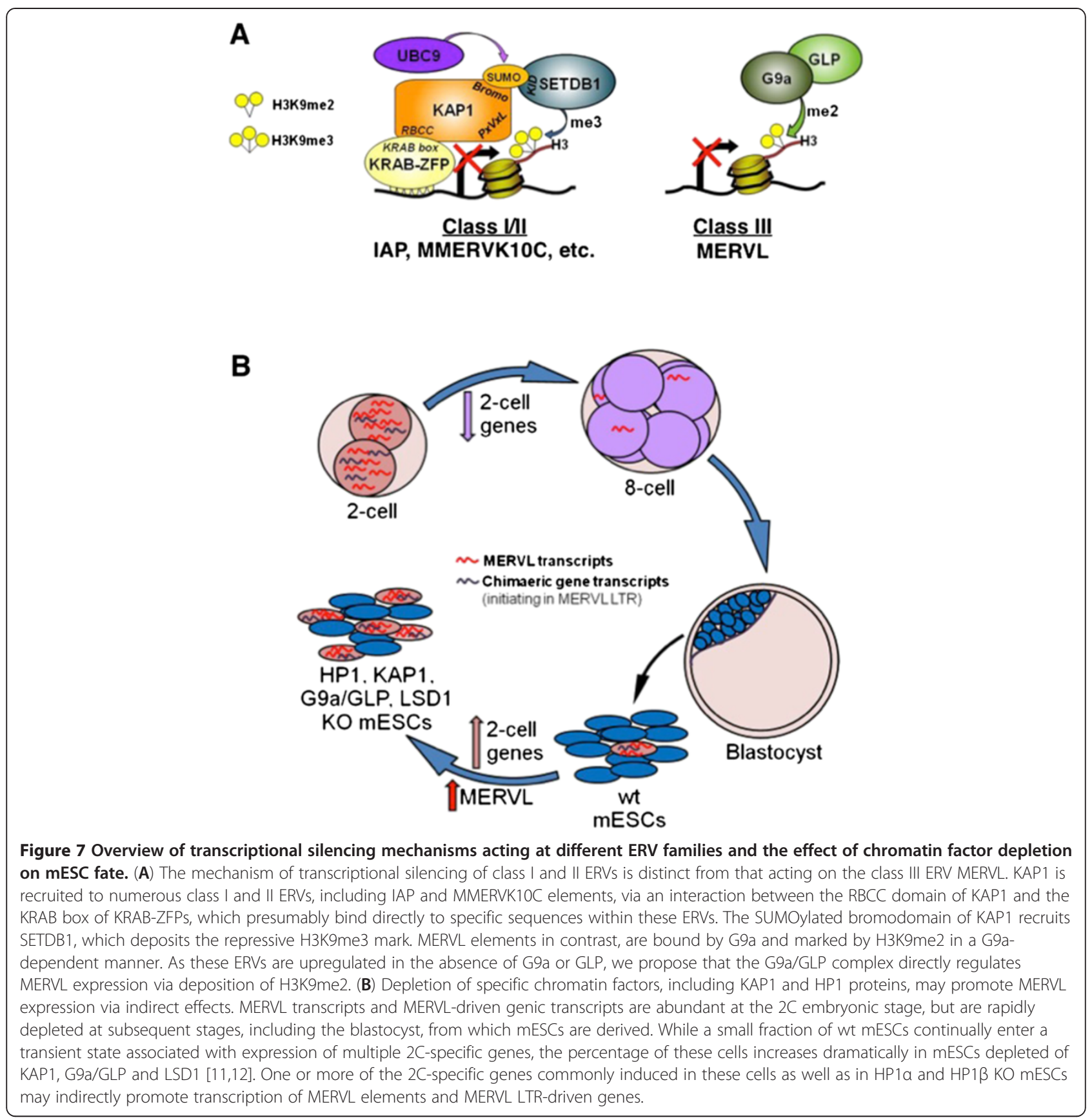

catalytic activity of G9a but not GLP. As these elements are bound by G9a and marked by $\mathrm{H} 3 \mathrm{~K} 9 \mathrm{me} 2$ in a G9adependent manner, we propose that the G9a/GLP complex acts directly at MERVL ERVs to maintain these elements in a silent state (Figure 7A). In contrast to IAP ERVs, MERVL ERVs show significantly higher levels of H3K9me2 than H3K9me3 enrichment. Taken together with the observation that HP1 $\alpha$ and HP1 $\beta$ are detected at IAP but not MERVL ERVs, our results indicate that these H3K9 methylation 'readers' bind preferentially to $\mathrm{H} 3 \mathrm{~K} 9 \mathrm{me} 3$ marked regions in vivo, though they are apparently not required for SETDB1-dependent silencing [25].

Although the molecular basis of H3K9me2-mediated transcriptional repression of MERVL elements remains to be determined, we propose that this pathway is particularly important in early embryogenesis. Consistent with this model, G9a and Glp mRNA levels are relatively low in the $2 \mathrm{C}$ embryo (Figure $\mathrm{S} 3 \mathrm{C}$ ) and $\mathrm{H} 3 \mathrm{~K} 9 \mathrm{me} 2$ is depleted on the paternal genome at this stage [65-67], perhaps explaining why MERVL elements and zygotic genes 
driven by the MERVL promoters are expressed at the $2 \mathrm{C}$ stage and silenced shortly thereafter $[3,10]$.

Intriguingly, depletion of several chromatin factors in mESCs including HP1 $\alpha$ and HP1 $\beta$ (this study), G9a/GLP (this study and [12]), LSD1 [11], KAP1 [28], RYBP [68] and ZFP42 (REX1) [69], leads to upregulation of MERVL elements. Depletion of a subset of these factors, including KAP1, G9a, LSD1 and RYBP, also leads to derepression of genes highly expressed in preimplantation embryos, such as Zfp352 and Zscan4 [12,68]. Here, we show that the same is true for HP1 $\alpha$ and HP1 $1 \beta$ KO mESCs. Derepression of MERVL elements in mESCs deficient in each of these chromatin factors may be due at least in part to an increase in the number of cells expressing $2 \mathrm{C}$-specific genes, which may in turn stimulate MERVL expression (Figure 7B).

Complicating interpretation of these observations is the fact that a significant number of genes expressed specifically at the $2 \mathrm{C}$ stage initiate transcription within MERVL LTRs, such as Zfp352 [53] and Tdpoz4 [55], or are located within a few kb of a MERVL LTR [11,12]. Thus, it is not clear whether induction of a subset of the 2C-specific genes in the above mentioned KO mESCs induce MERVL expression as a 'secondary effect', or whether aberrant genic transcription of $2 \mathrm{C}$ genes in these $\mathrm{KO}$ lines results predominantly as a direct consequence of perturbation of MERVL silencing per se. These alternative explanations are not necessarily mutually exclusive.

\section{Conclusions}

In summary, our results indicate that G9a and GLP play a direct role in silencing of MERVL ERVs and genes driven by MERVL LTR promoters via G9a-mediated deposition of H3K9me2, while the KAP1-interacting H3K9 KMTase SETDB1 is neither recruited to MERVL elements nor required for their repression. Conversely, the results presented here are consistent with the model that induction of MERVL expression following deletion of KAP1, HP1 $\alpha$ and HP1 $\beta$ occurs primarily via indirect effects. Given that expression of MERVL elements, unlike other ERVs, is normally restricted to the $2 \mathrm{C}$ embryo, any perturbation of mESCs that induces a nuclear milieu permissive for expression of $2 \mathrm{C}$-specific genes may indirectly induce expression of these ERVs as well as genes driven by MERVL promoters.

\section{Methods}

\section{Cell culture, RNA isolation, qRT-PCR}

Mouse ESC lines, including HP1 $\alpha$ and HP1 $\beta$ KOs and their corresponding wt line HM1 [25] and G9a and GLP KOs and their corresponding wt line TT2 [70] were cultured in DMEM supplemented with 15\% FBS (Thermo Scientific HyClone, Logan, UT, USA), 20 mM HEPES, $0.1 \mathrm{mM}$ nonessential amino acids, $0.1 \mathrm{mM}$ 2-mercaptoethanol, $100 \mathrm{U} /$ $\mathrm{ml}$ penicillin, $0.05 \mathrm{mM}$ streptomycin, leukemia-inhibitory factor and $2 \mathrm{mM} \mathrm{L}$-glutamine on gelatinized plates. RNA was isolated using GenElute mRNA miniprep kit (SigmaAldrich, St Louis, MO, USA) and reverse transcribed using SuperScript III (Invitrogen, Carlsbad, CA, USA) as per the manufacturer's instructions. Quantitative RT-PCR was carried out using SsoFAST ${ }^{\text {"m }}$ EvaGreen Supermix (Bio-Rad, Hercules, CA, USA) on StepOne" Software v2.1 (Applied Biosystems, Foster City, CA, USA). Data are presented as mean $+/$ - standard deviations of three technical replicates. Primer efficiencies were 95 to 105\%. Dissociation curve analysis was performed after the end of the PCR to confirm the presence of a single and specific product. Corresponding ERV primers detect 519 MERVL elements, 202 MMERVK10C elements and 638 IAPEz elements. Primer sequences are listed in Additional file 3.

\section{RNAi}

For RNA collection, 7,000 mESCs per well of a 96-well plate were plated into antibiotic-free ES medium the day before transfection. Transfection was performed according to the manufacturer's protocol, using $100 \mathrm{nM}$ of each siRNA (Dharmacon siGENOME SMARTpool) and $0.4 \mu \mathrm{l}$ DharmaFECT 1 reagent per well (Thermo Scientific Dharmacon, Lafayette, CO, USA). The first day after transfection, a fraction of cells was transferred into a 12-well plate into antibiotic-free ES medium, and the transfection was repeated on the third day. The following day (approximately $30 \mathrm{~h}$ ) after the second $\mathrm{KD}$, most of the cells were collected for RNA for confirmation of KD efficiency (day 1 after the second $\mathrm{KD}$ ), and the rest were plated onto $3.5-\mathrm{cm}$ dishes for expansion and collection of RNA to monitor ERV derepression on day 4 after the second KD. For ChIP on day 4 after the second KD, the same steps were performed accounting for the increased volumes, with the cells plated onto a 12-well plate for the first transfection, transferred onto two $6 \mathrm{~cm}$ dishes for the second transfection and onto 4 to $6 \times 10 \mathrm{~cm}$ dishes for collection on day 4 post second transfection, with approximately $2 \times 10^{5}$ cells saved at day $1(30 \mathrm{~h})$ for the RNA analysis of KD efficiency.

\section{Native ChIP (N-ChIP) and cross-linked ChIP}

For N-ChIP, $1 \times 10^{7} \mathrm{ES}$ cells for each cell line were resuspended in douncing buffer $(10 \mathrm{mM}$ Tris- $\mathrm{HCl}$ $\mathrm{pH}$ 7.5, $4 \mathrm{mM} \mathrm{MgCl} 2,1 \mathrm{mM} \mathrm{CaCl}$ ) and homogenized through a $25 \mathrm{G} 5 / 8$ needle syringe for 20 repetitions. Following addition of $1.25 \mu \mathrm{l}(50 \mathrm{U} / \mu \mathrm{l})$ of MNase (Worthington Biochemicals Corp., Lakewood Township, $\mathrm{NJ}$, USA), the sample was incubated at $37^{\circ} \mathrm{C}$ for $7 \mathrm{~min}$. The reaction was quenched with $0.5 \mathrm{M}$ EDTA and incubated on ice for $5 \mathrm{~min}$. $1 \mathrm{ml}$ of hypotonic buffer (0.2 mM EDTA pH 8.0, $0.1 \mathrm{mM}$ benzamidine, $0.1 \mathrm{mM}$ PMSF, $1.5 \mathrm{mM}$ DTT) was added and the sample incubated on ice for $1 \mathrm{~h}$. Cellular debris was pelleted and the supernatant was recovered. Protein A (Millipore, 16- 
156) and G Sepharose (Millipore, 16-266) (Millipore, Billerica, MA, USA) beads were blocked with singlestranded salmon sperm DNA and BSA, washed and resuspended in IP buffer (10 mM Tris- $\mathrm{HCl}(\mathrm{pH} 8.0), 1 \%$ Triton X-100, 0.10\% deoxycholate, $0.10 \%$ SDS, $90 \mathrm{mM}$ $\mathrm{NaCl}, 2 \mathrm{mM}$ EDTA). Blocked protein A/G beads were added to the digested chromatin fractions and rotated at $4^{\circ} \mathrm{C}$ for $2 \mathrm{~h}$ to preclear chromatin. An aliquot of a $100 \mu \mathrm{l}$ of precleared chromatin was purified by phenolchloroform extraction, and DNA fragment sizes were analyzed and confirmed to correspond to 1 to 3 nucleosome fragments. Chromatin was subdivided into aliquots for each IP. Antibodies specific for unmodified H3 (5 $\mathrm{Hl}$, Sigma-Aldrich H9289), H3K9me1 (5 ul, Abcam ab8896;), H3K9me2 (5 $\mu \mathrm{l}$, Abcam ab1220) (Abcam, Cambridge, UK), H3K9me3 (5 $\mu$ l, Active Motif 39161) (Active Motif, Carlsbad, CA, USA), and control IgG (1 $\mu$ l, Sigma-Aldrich I8140) were added to each tube and rotated at $4^{\circ} \mathrm{C}$ for 1 hour. The antibody-protein-DNA complex was precipitated by adding $20 \mu \mathrm{l}$ of the blocked protein A/G beads and rotated at $4^{\circ} \mathrm{C}$ overnight $(\mathrm{O} / \mathrm{N})$. The complex was washed and eluted; IP'd material was purified using the QIAquick PCR Purification Kit (Qiagen, Venlo, Netherlands).

Cross-link ChIP for HP1 proteins was performed as described by Metivier et al. [71]. Briefly, $2 \times 10^{7} \mathrm{ES}$ cells were harvested. Chromatin was cross-linked in $10 \mathrm{ml}$ of PBS $+1 \%$ formaldehyde for $10 \mathrm{~min}$ at room temperature (RT). Reaction was quenched with $0.125 \mathrm{M}$ glycine and incubated at RT for $5 \mathrm{~min}$. Cells were washed two times with PBS and resuspended in $1 \mathrm{ml}$ of collection buffer. The solution was incubated on ice for $10 \mathrm{~min}$, then for $10 \mathrm{~min}$ at $30^{\circ} \mathrm{C}$. Cells were lysed by vortexing pellets sequentially in $1 \mathrm{ml}$ buffer A, $1 \mathrm{ml}$ buffer B, followed by incubation of cell pellets at RT in $1 \mathrm{ml}$ of lysis buffer for 10 min. Chromatin was sonicated using the Diagenode Bioruptor (Diagenode, Liege, Belgium) at high setting for $18 \mathrm{~min}$ at intervals of $30 \mathrm{sec}$ 'on' and $30 \mathrm{sec}$ 'off' to achieve fragment lengths of 250 to $500 \mathrm{bp}$. For each IP, 2 $\times 10^{6}$ cell-equivalents of precleared chromatin were used and diluted 2.5 times in IP buffer. Antibodies specific for HP1 $\alpha$ (10 $\mu \mathrm{l}$, academic, S. Smale), HP1 $\beta$ (5 $\mu \mathrm{l}$, NEB, HP1beta (D2F2) XP $\mathrm{P}^{\mathrm{Tm}}$ rabbit mAb 8676) and control IgG ( $1 \mu$ l, Sigma-Aldrich I8140) were added into each IP and rotated at $4^{\circ} \mathrm{C} \mathrm{O} / \mathrm{N}$. To each tube, $40 \mathrm{ul}$ of precleared beads were added, and tubes were rotated at $4^{\circ} \mathrm{C}$ for $2 \mathrm{~h}$. The complex was washed and eluted off the beads. The cross-links were reversed $\mathrm{O} / \mathrm{N}$ at $65^{\circ} \mathrm{C}$, and the DNA was treated with Proteinase $\mathrm{K}$ and RNase A. DNA was purified using the Qiaquick PCR Purification Kit (Qiagen) and analyzed by qPCR with respect to input using SsoFAST ${ }^{\mathrm{TM}}$ EvaGreen Supermix (Bio-Rad). Primer sequences are listed in Additional file 3.

Cross-linked ChIP for G9a and KAP1 were performed similarly with several modifications. Approximately $5 \times 10^{6}$
TT2 or G9a KO mESCs were harvested cross-linked in $0.75 \%$ formaldehyde in PBS for 10 min, quenched with glycine, and lysed in ice-cold radioimmunoprecipitation assay buffer (RIPA) (50 mM Tris pH 8.0, $150 \mathrm{mM} \mathrm{NaCl}, 1 \% \mathrm{NP}-$ $40,0.25 \%$ sodium deoxycholate, $0.1 \%$ SDS). Chromatin was sonicated with the Bioruptor on $30 \mathrm{sec}$ 'on' and $30 \mathrm{sec}$ 'off' to achieve 200 to $600 \mathrm{bp}$ fragment size for each sample. The samples were divided into three equal aliquots and incubated $\mathrm{O} / \mathrm{N}$ at $4^{\circ} \mathrm{C}$ with $4 \mu \mathrm{g}$ of purified mouse IgG (Sigma-Aldrich), mouse monoclonal anti-G9a (R\&D Systems PP-A8620-00) (R\&D Systems, Minneapolis, MN, USA), or mouse monoclonal anti-KAP1 (Abcam Ab22553). Samples were precipitated with $30 \mu$ protein-G Dynabeads (Invitrogen), washed three times with ice-cold RIPA buffer and eluted by shaking in $0.1 \mathrm{M} \mathrm{NaHCO}_{3}, 1 \% \mathrm{SDS}, 20 \mathrm{mM}$ DTT for $15 \mathrm{~min}$. Cross-links were reversed by heating samples at $95^{\circ} \mathrm{C}$ for $5 \mathrm{~min}$ in the presence of $300 \mathrm{mM} \mathrm{NaCl}$. DNA was RNase A-treated, purified and qPCR was performed as described above. Primers used or ChIP-qPCR analyses detect: 638 IAP ERVs, 519 MERV-L ERVs, 202 MMERVK10C ERVs, 637 MERV-L pol internal regions, as determined by in silico PCR on the UCSC genome browser.

\section{RNA-seq, data normalization and Z-score calculation}

RNA-seq libraries were constructed from mRNA as described in Morin et al. [72] from $10 \mu \mathrm{g}$ of DNaseItreated total RNA, and 75 bp paired-end sequencing was performed on an Illumina Genome Analyzer following the recommended protocol (Illumina Inc., Hayward, CA, USA). Sequence reads were aligned to the mouse reference genome (mm9) using BWA v0.5.9 [73] with SmithWaterman alignment disabled and annotated exon-exon junctions compiled from Ensembl [74], RefSeq [75] and UCSC [76] (downloaded from http://genome.ucsc.edu on 17 August 2011). To quantify expression levels and the strength of KAP1 and SETDB1 marks, we calculated reads per kilobase per million mapped reads (RPKM) $[77,78]$ for genomic regions of interest. For pair-wise sample comparisons, an empirical Z-score was calculated assuming the distribution of RPKMs for each sample followed a Poisson model:

$$
Z \text {-score }=\left(R P K M_{A}-R P K M_{B}\right) / \sqrt{\left(R P K M_{A}-r_{A B} R P K M_{B}\right)}
$$

where $R P K M_{A}$ and $R P K M_{B}$ are RPKMs in the region of interest of $A$ and $B$ samples respectively, and $r_{A B}=N_{A}$ / $\mathrm{N}_{\mathrm{B}}$, where $N x$ is the total number of aligned reads used for normalization.

\section{KAP1 ChIP-seq data analysis}

In order to compare the coverage of KAP1 and SETDB1 among all families of ERVs and generate the average density of KAP1 in the genomic regions flanking ERVs, we mined the published KAP1 ChIP-seq data set [46]. Sequence reads 
for KAP1 were remapped to mm9 (NCBI 37) using BWA v0.5.9 [79] and default parameters. Reads having identical coordinates were collapsed into a single read, and reads with mapQ > =10 passed to FindPeaks 3.1 [80] (with a fixed directional read extension of $300 \mathrm{bp}$ ) for generation of an unthresholded coverage WIG file to be visualized in the UCSC genome browser [81]. This coverage file was used to calculate KAP1 RPKM values for various regions of interest. Subsequently, we identified all enriched regions with a peak-height $\geq 10$ and generated a thresholded coverage WIG file for KAP1, using FindPeaks. This WIG file was used to generate the profiles at the genomic regions flanking ERVs.

The KAP1 profile was generated at the genomic flanks of intact elements (flanked by two identical LTRs), which satisfied the length criteria, for three ERV families: MMERVK10C, IAPEz and MERVL. The mean density of KAP1 for each family was calculated for $50 \mathrm{bp}$ bins within $6 \mathrm{~kb}$ distance at $5^{\prime}$ and 3 ' flanks of elements by agglomerating the coverage inside the bins for all elements of one family and dividing this number by the number of elements and total number of aligned reads in the KAP1 IP.

\section{Detection of chimeric transcripts}

Chimeric transcripts containing ERV and genic sequences were identified by exploiting the genomic locations of paired-end reads. Mate-pair reads separated by more than one standard deviation from the mean fragment size were identified, and those mate-pairs containing one read in an ERV located upstream of the first exon of a gene, and the other read in an annotated genic exon of that gene, were enumerated. The number of chimeric mate-pairs was calculated for each chimeric transcript and the transcripts with the number of chimeric mate-pairs $>5$ were scored as valid transcripts.

\section{Immunoprecipitation and Western blotting}

Whole-cell extracts were prepared by lysing cells in modified RIPA buffer (50 mM Tris pH 8.0, $150 \mathrm{mM} \mathrm{NaCl}, 1 \%$ NP-40, $0.25 \%$ deoxycholate, $0.1 \%$ SDS). Nuclear extracts were prepared as previously described [82] except nuclei were extracted for $30 \mathrm{~min}$ in $20 \mathrm{mM}$ HEPES $\mathrm{pH}$ 7.9, $500 \mathrm{mM} \mathrm{KCl}, 1.5 \mathrm{mM} \mathrm{MgCl} 2,25 \%$ glycerol. Protein concentrations were determined by Bradford assay (Bio-Rad) using BSA as a standard. For immunoprecipitation, $200 \mu \mathrm{g}$ of nuclear extract was diluted to approximately $170 \mathrm{mM}$ $\mathrm{KCl}$ incubated with $1 \mu \mathrm{g}$ of mouse monoclonal anti-KAP1 (Abcam ab22553), mouse monoclonal anti-G9a (R\&D Systems PP-A8620-00), or mouse IgG (Sigma-Aldrich) and samples were rotated overnight at $4^{\circ} \mathrm{C}$. Complexes were precipitated with protein G-Dynabeads (Invitrogen), washed three times with ice-cold wash buffer $(20 \mathrm{mM}$ HEPES pH 7.9, $200 \mathrm{mM} \mathrm{KCl,} \mathrm{1 \%} \mathrm{NP-40,} \mathrm{10 \%} \mathrm{glycerol)} \mathrm{and}$ eluted by boiling beads in SDS-PAGE sample buffer containing DTT. For Western blot analysis, protein extracts or immunoprecipitated samples were separated on $7.5 \%$ or $10 \%$ SDS-PAGE gels, transferred to nitrocellulose membranes, blocked with $4 \%$ skim milk in Tris-buffered saline (TBS: $20 \mathrm{mM}$ Tris- $\mathrm{HCl} \mathrm{pH}$ 7.5, $100 \mathrm{mM} \mathrm{NaCl}$ ) and probed overnight at $4^{\circ} \mathrm{C}$ with primary antibodies diluted in TBS containing 0.1\% Tween-20 (TBS-T): 1:5000 mouse antiKAP1 (Abcam), 1:1000 mouse anti-G9a (R\&D Systems), 1:1000 mouse anti-GLP (R\&D Systems PP-B0422-00), 1:2000 rabbit anti-LSD1 (Abcam ab17721), 1:500 rabbit anti-HP1 $\beta$ (Cell Signaling 2613) (Cell Signaling Technology, Danvers, MA, USA) and 1:500 rabbit anti-Pol II large subunit (Santa Cruz Biotechnology sc-899) (Santa Cruz Biotechnology, Santa Cruz, CA, USA). Blots were subsequently washed in TBS-T, incubated in IRDye-conjugated secondary antibodies diluted 1:20,000 in 2\% milk in TBS-T washed again in TBS-T and scanned on the Odyssey infrared imaging system (LI-COR Biosciences, Lincoln, ME, USA).

\section{Availability of supporting data}

The data sets supporting the results of this article are available in NCBI's Gene Expression Omnibus repository [83], GEO Series accession number GSE47370 (http://www.ncbi. nlm.nih.gov/geo/query/acc.cgi?acc=GSE47370).

\section{Additional files}

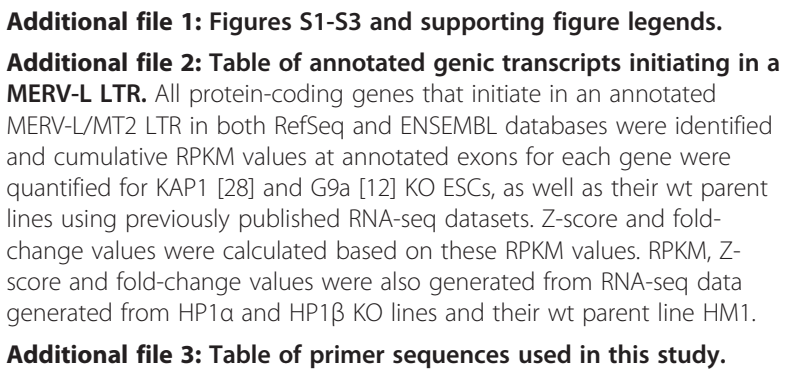

Competing interests

The authors declare that they have no competing interests.

\section{Authors' contributions}

IM performed most experiments, PT performed qRT-PCR of Zfp352, ChIPqPCR of G9a and KAP1 and Western blotting, PG performed ChIP-qPCR of HP1s, MK performed bioinformatics analyses, with support from SJ, PS contributed the HP1 KO lines, IM and ML designed the study and wrote the manuscript. All authors read and approved the final manuscript.

\section{Acknowledgements}

We thank Yoichi Shinkai for the G9a and GLP KO mESCs, Stephen Smale for the HP1a antibody, and Dixie Mager and Julie Brind'Amour for helpful discussions. We also thank Misha Bilenky for technical support. This work was supported by Canadian Institutes of Health Research (CIHR) grants 77805 and 92090 to ML. ML is a CIHR New Investigator. SJ is a Scholar of the Michael Smith Foundation for Health Research (MSFHR) and MK was supported by a MSFHR postdoctoral fellowship.

\section{Author details}

'Department of Medical Genetics, Life Sciences Institute, 2350 Health Sciences Mall, University of British Columbia, Vancouver, British Columbia V6T 
1Z3, Canada. ${ }^{2}$ British Columbia Cancer Agency, Genome Sciences Centre, 675 West 10th Avenue, Vancouver, British Columbia V5Z 4S6, Canada.

${ }^{3}$ Fächereverbund Anatomie, Institut für Zell and Neurobiologie, Charite -

Universitätsmedizin, Charitéplatz 1, 10117, Berlin, Germany.

Received: 14 December 2012 Accepted: 8 May 2013

Published: 4 June 2013

\section{References}

1. Jern P, Sperber GO, Blomberg J: Use of endogenous retroviral sequences (ERVs) and structural markers for retroviral phylogenetic inference and taxonomy. Retrovirology 2005, 2:50.

2. Maksakova IA, Romanish MT, Gagnier L, Dunn CA, van de Lagemaat LN, Mager DL: Retroviral elements and their hosts: insertional mutagenesis in the mouse germ line. PLoS Genet 2006, 2:e2.

3. Peaston AE, Evsikov AV, Graber JH, de Vries WN, Holbrook AE, Solter D, Knowles BB: Retrotransposons regulate host genes in mouse oocytes and preimplantation embryos. Dev Cell 2004, 7:597-606.

4. International Mouse Genome Sequencing Consortium: Initial sequencing and comparative analysis of the mouse genome. Nature 2002, 420:520-562.

5. Kigami D, Minami N, Takayama H, Imai H: MuERV-L is one of the earliest transcribed genes in mouse one-cell embryos. Biol Reprod 2003, 68:651-654.

6. Svoboda P, Stein P, Anger M, Bernstein E, Hannon GJ, Schultz RM: RNAi and expression of retrotransposons MuERV-L and IAP in preimplantation mouse embryos. Dev Biol 2004, 269:276-285.

7. Ribet D, Louvet-Vallee S, Harper F, de Parseval N, Dewannieux M, Heidmann O, Pierron G, Maro B, Heidmann T: MuERV-L is the progenitor of the "orphan" Epsilon virus-like particles of the early mouse embryo. J Virol 2008, 82:1622-1625.

8. Cohen CJ, Lock WM, Mager DL: Endogenous retroviral LTRs as promoters for human genes: a critical assessment. Gene 2009, 448:105-114

9. Rebollo R, Romanish MT, Mager DL: Transposable elements: an abundant and natural source of regulatory sequences for host genes. Annu Rev Genet 2012, 42:21-42.

10. Evsikov AV, de Vries WN, Peaston AE, Radford EE, Fancher KS, Chen FH, Blake JA, Bult CJ, Latham KE, Solter D, Knowles BB: Systems biology of the 2-cell mouse embryo. Cytogenet Genome Res 2004, 105:240-250.

11. Macfarlan TS, Gifford WD, Agarwal S, Driscoll S, Lettieri K, Wang J, Andrews SE, Franco L, Rosenfeld MG, Ren B, Pfaff SL: Endogenous retroviruses and neighboring genes are coordinately repressed by LSD1/KDM1A. Genes Dev 2011, 25:594-607.

12. Macfarlan TS, Gifford WD, Driscoll S, Lettieri K, Rowe HM, Bonanomi D, Firth A, Singer O, Trono D, Pfaff SL: Embryonic stem cell potency fluctuates with endogenous retrovirus activity. Nature 2012, 487:57-63.

13. Howard G, Eiges R, Gaudet F, Jaenisch R, Eden A: Activation and transposition of endogenous retroviral elements in hypomethylation induced tumors in mice. Oncogene 2008, 27:404-408.

14. Jaenisch $\mathrm{R}$, Schnieke A, Harbers K: Treatment of mice with 5-azacytidine efficiently activates silent retroviral genomes in different tissues. Proc Natl Acad Sci USA 1985, 82:1451-1455.

15. Walsh CP, Chaillet JR, Bestor TH: Transcription of IAP endogenous retroviruses is constrained by cytosine methylation. Nat Genet 1998, 20:116-117.

16. Gaudet F, Hodgson JG, Eden A, Jackson-Grusby L, Dausman J, Gray JW Leonhardt $H$, Jaenisch R: Induction of tumors in mice by genomic hypomethylation. Science 2003, 300:489-492.

17. Dodge JE, Okano M, Dick F, Tsujimoto N, Chen T, Wang S, Ueda Y, Dyson N, Li E: Inactivation of Dnmt3b in mouse embryonic fibroblasts results in DNA hypomethylation, chromosomal instability, and spontaneous immortalization. J Biol Chem 2005, 280:17986-17991.

18. Bourc'his D, Bestor TH: Meiotic catastrophe and retrotransposon reactivation in male germ cells lacking Dnmt3L. Nature 2004, 431:96-99.

19. Hata K, Kusumi M, Yokomine T, Li E, Sasaki H: Meiotic and epigenetic aberrations in Dnmt3L-deficient male germ cells. Mol Reprod Dev 2006, 73:116-122.

20. Dong KB, Maksakova IA, Mohn F, Leung D, Appanah R, Lee S, Yang HW, Lam LL, Mager DL, Schübeler D, Tachibana M, Shinkai Y, Lorincz MC: DNA methylation in ES cells requires the lysine methyltransferase G9a but not its catalytic activity. EMBO J 2008, 27:2691-2701.

21. Hutnick LK, Huang X, Loo TC, Ma Z, Fan G: Repression of retrotransposal elements in mouse embryonic stem cells is primarily mediated by a DNA methylation-independent mechanism. J Biol Chem 2010, 285:21082-21091.
22. Damelin M, Bestor TH: Biological functions of DNA methyltransferase 1 require its methyltransferase activity. Mol Cell Biol 2007, 27:3891-3899.

23. Karimi MM, Goyal P, Maksakova Irina A, Bilenky M, Leung D, Tang Jie X, Shinkai Y, Mager Dixie L, Jones S, Hirst M, Lorincz Matthew C: DNA methylation and SETDB1/H3K9me3 regulate predominantly distinct sets of genes, retroelements, and chimeric transcripts in mESCs. Cell Stem Cell 2011, 8:676-687

24. Matsui T, Leung D, Miyashita H, Maksakova IA, Miyachi H, Kimura H, Tachibana M, Lorincz MC, Shinkai Y: Proviral silencing in embryonic stem cells requires the histone methyltransferase ESET. Nature 2010, 464:927-931.

25. Maksakova IA, Goyal P, Bullwinkel J, Brown JP, Bilenky M, Mager DL, Singh $\mathrm{PB}$, Lorincz MC: H3K9me3 binding proteins are dispensable for SETDB1/ H3K9me3-dependent retroviral silencing. Epigenetics Chromatin 2011, 4:12.

26. Ivanov AV, Peng H, Yurchenko V, Yap KL, Negorev DG, Schultz DC, Psulkowski E, Fredericks WJ, White DE, Maul GG, Sadofsky MJ, Zhou MM, Rauscher FJ 3rd: PHD domain-mediated E3 ligase activity directs intramolecular sumoylation of an adjacent bromodomain required for gene silencing. Mol Cell 2007, 28:823-837.

27. Wolf D, Goff SP: Embryonic stem cells use ZFP809 to silence retroviral DNAs. Nature 2009, 458:1201-1204.

28. Rowe HM, Jakobsson J, Mesnard D, Rougemont J, Reynard S, Aktas T, Maillard PV, Layard-Liesching H, Verp S, Marquis J, Spitz F, Constam DB, Trono D: KAP1 controls endogenous retroviruses in embryonic stem cells. Nature 2010, 463:237-240

29. Sripathy SP, Stevens J, Schultz DC: The KAP1 corepressor functions to coordinate the assembly of de novo HP1-demarcated microenvironments of heterochromatin required for KRAB zinc finger protein-mediated transcriptional repression. Mo/ Cell Biol 2006, 26:8623-8638.

30. Friedman JR, Fredericks WJ, Jensen DE, Speicher DW, Huang XP, Neilson EG, Rauscher FJ 3rd: KAP-1, a novel corepressor for the highly conserved KRAB repression domain. Genes Dev 1996, 10:2067-2078.

31. Vermaak D, Malik HS: Multiple roles for heterochromatin protein 1 genes in Drosophila. Annu Rev Genet 2009, 43:467-492.

32. Kwon SH, Workman JL: The changing faces of HP1: From heterochromatin formation and gene silencing to euchromatic gene expression: HP1 acts as a positive regulator of transcription. Bioessays 2011, 33:280-289.

33. Singh PB, Georgatos SD: HP1: facts, open questions, and speculation. J Struct Biol 2002, 140:10-16.

34. Ryan RF, Schultz DC, Ayyanathan K, Singh PB, Friedman JR, Fredericks WJ, Rauscher FJ 3rd: KAP-1 corepressor protein interacts and colocalizes with heterochromatic and euchromatic HP1 proteins: a potential role for Kruppel-associated box-zinc finger proteins in heterochromatinmediated gene silencing. Mol Cell Biol 1999, 19:4366-4378.

35. Bannister AJ, Zegerman P, Partridge JF, Miska EA, Thomas JO, Allshire RC, Kouzarides T: Selective recognition of methylated lysine 9 on histone $\mathrm{H} 3$ by the HP1 chromo domain. Nature 2001, 410:120-124.

36. Lachner M, O'Carroll D, Rea S, Mechtler $K$, Jenuwein T: Methylation of histone H3 lysine 9 creates a binding site for HP1 proteins. Nature 2001, 410:116-120.

37. Nielsen AL, Oulad-Abdelghani M, Ortiz JA, Remboutsika E, Chambon P, Losson R: Heterochromatin formation in mammalian cells: interaction between histones and HP1 proteins. Mol Cell 2001, 7:729-739.

38. Groner AC, Meylan S, Ciuffi A, Zangger N, Ambrosini G, Denervaud N, Bucher $P$, Trono D: KRAB-zinc finger proteins and KAP1 can mediate long-range transcriptional repression through heterochromatin spreading. PLoS Genet 2010, 6:e1000869.

39. Singh PB: HP1 proteins - what is the essential interaction? Genetika 2010 46:1424-1429.

40. Lechner MS, Begg GE, Speicher DW, Rauscher FJ 3rd: Molecular determinants for targeting heterochromatin protein 1-mediated gene silencing: direct chromoshadow domain-KAP-1 corepressor interaction is essential. Mol Cell Biol 2000, 20:6449-6465.

41. Thiru A, Nietlispach D, Mott HR, Okuwaki M, Lyon D, Nielsen PR, Hirshberg M, Verreault A, Murzina NV, Laue ED: Structural basis of HP1/PXVXL motif peptide interactions and HP1 localisation to heterochromatin. EMBO 2004, 23:489-499.

42. Riclet R, Chendeb M, Vonesch JL, Koczan D, Thiesen HJ, Losson R, Cammas F: Disruption of the interaction between TIF1beta and HP1 leads to a switch from DNA hyper- to hypomethylation and H3K9 to H3K27 trimethylation on the MEST promoter correlating with gene reactivation. Mol Biol Cell 2008, 20:296-305. 
43. Nielsen AL, Ortiz JA, You J, Oulad-Abdelghani M, Khechumian R, Gansmuller A, Chambon P, Losson R: Interaction with members of the heterochromatin protein 1 (HP1) family and histone deacetylation are differentially involved in transcriptional silencing by members of the TIF1 family. EMBO J 1999, 18:6385-6395.

44. Schultz DC, Ayyanathan K, Negorev D, Maul GG, Rauscher FJ 3rd: SETDB1: a novel KAP-1-associated histone $\mathrm{H3}$, lysine 9-specific methyltransferase that contributes to HP1-mediated silencing of euchromatic genes by KRAB zinc-finger proteins. Genes Dev 2002, 16:919-932.

45. Reichmann J, Crichton JH, Madej MJ, Taggart M, Gautier P, Garcia-Perez JL, Meehan RR, Adams IR: Microarray analysis of LTR retrotransposon silencing identifies $\mathrm{Hdac} 1$ as a regulator of retrotransposon expression in mouse embryonic stem cells. PLoS Comput Biol 2012, 8:e1002486.

46. Quenneville S, Verde G, Corsinotti A, Kapopoulou A, Jakobsson J, Offner S, Baglivo I, Pedone Paolo V, Grimaldi G, Riccio A, Trono D: In embryonic stem cells, ZFP57/ KAP1 recognize a methylated hexanucleotide to affect chromatin and DNA methylation of imprinting control regions. Mol Cell 2011, 44:361-372.

47. Yuan P, Han J, Guo G, Orlov YL, Huss M, Loh YH, Yaw LP, Robson P, Lim B, $\mathrm{Ng} \mathrm{HH}$ : Eset partners with Oct4 to restrict extraembryonic trophoblast lineage potential in embryonic stem cells. Genes Dev 2009, 23:2507-2520

48. Rebollo R, Karimi MM, Bilenky M, Gagnier L, Miceli-Royer K, Zhang Y, Goyal P, Keane TM, Jones S, Hirst M, Lorincz MC, Mager DL: Retrotransposoninduced heterochromatin spreading in the mouse revealed by insertional polymorphisms. PLoS Genet 2011, 7:e1002301

49. Tachibana M, Sugimoto K, Nozaki M, Ueda J, Ohta T, Ohki M, Fukuda M, Takeda N, Niida H, Kato H, Shinkai Y: G9a histone methyltransferase plays a dominant role in euchromatic histone $\mathrm{H} 3$ lysine 9 methylation and is essential for early embryogenesis. Genes Dev 2002, 16:1779-1791.

50. Tachibana M, Matsumura Y, Fukuda M, Kimura H, Shinkai Y: G9a/GLP complexes independently mediate $\mathrm{H} 3 \mathrm{~K} 9$ and DNA methylation to silence transcription. EMBO J 2008, 27:2681-2690.

51. Chin HG, Esteve PO, Pradhan M, Benner J, Patnaik D, Carey MF, Pradhan S: Automethylation of G9a and its implication in wider substrate specificity and HP1 binding. Nucleic Acids Res 2007, 35:7313-7323.

52. Tang F, Barbacioru C, Nordman E, Bao S, Lee C, Wang X, Tuch BB, Heard E, Lao K, Surani MA: Deterministic and stochastic allele specific gene expression in single mouse blastomeres. PLoS One 2011, 6:e21208.

53. Liu TY, Chen HH, Lee KH, Choo KB: Display of different modes of transcription by the promoters of an early embryonic gene, Zfp352, in preimplantation embryos and in somatic cells. Mol Reprod Dev 2003, 64:52-60.

54. Falco G, Lee SL, Stanghellini I, Bassey UC, Hamatani T, Ko MS: Zscan4: a novel gene expressed exclusively in late 2-cell embryos and embryonic stem cells. Dev Biol 2007, 307:539-550.

55. Huang C-J, Chen C-Y, Chen H-H, Tsai S-F, Choo K-B: TDPOZ, a family of bipartite animal and plant proteins that contain the TRAF (TD) and POZ/ BTB domains. Gene 2004, 324:117-127.

56. Benit L, Lallemand J-B, Casella J-F, Philippe H, Heidmann T: ERV-L elements: a family of endogenous retrovirus-like elements active throughout the evolution of mammals. J Virol 1999, 73:3301-3308.

57. Taruscio D, Mantovani A: Factors regulating endogenous retroviral sequences in human and mouse. Cytogenet Genome Res 2004, 105:351-362.

58. Goff SP: Retrovirus restriction factors. Mol Cell 2004, 16:849-859.

59. Rowe HM, Trono D: Dynamic control of endogenous retroviruses during development. Virology 2011, 411:273-287.

60. Leung DC, Lorincz MC: Silencing of endogenous retroviruses: when and why do histone marks predominate? Trends Biochem Sci 2011, 37:127-133.

61. Wolf D, Goff SP: TRIM28 mediates primer binding site-targeted silencing of murine leukemia virus in embryonic cells. Cell 2007, 131:46-57.

62. Ravasi T, Huber T, Zavolan M, Forrest A, Gaasterland T, Grimmond S, Hume DA: Systematic characterization of the zinc-finger-containing proteins in the mouse transcriptome. Genome Res 2003, 13:1430-1442.

63. Urrutia R: KRAB-containing zinc-finger repressor proteins. Genome Biol 2003, 4:231.

64. Thomas $\mathrm{JH}$, Schneider S: Coevolution of retroelements and tandem zinc finger genes. Genome Res 2011, 21:1800-1812.

65. Lepikhov K, Walter J: Differential dynamics of histone $\mathrm{H} 3$ methylation at positions K4 and K9 in the mouse zygote. BMC Dev Biol 2004, 4:12.

66. Yeo S, Lee KK, Han YM, Kang YK: Methylation changes of lysine 9 of histone H3 during preimplantation mouse development. Mol Cells 2005, 20:423-428.

67. Santos F, Peters AH, Otte AP, Reik W, Dean W: Dynamic chromatin modifications characterise the first cell cycle in mouse embryos. Dev Biol 2005, 280:225-236.
68. Hisada K, Sanchez C, Endo TA, Endoh M, Roman-Trufero M, Sharif J, Koseki $H$, Vidal M: RYBP represses endogenous retroviruses and preimplantation- and germ line-specific genes in mouse embryonic stem cells. Mol Cell Biol 2012, 32:1139-1149.

69. Guallar D, Pérez-Palacios R, Climent M, Martínez-Abadía I, Larraga A, Fernández-Juan M, Vallejo C, Muniesa P, Schoorlemmer J: Expression of endogenous retroviruses is negatively regulated by the pluripotency marker Rex1/Zfp42. Nucleic Acids Res 2012, 40:8993-9007.

70. Tachibana M, Ueda J, Fukuda M, Takeda N, Ohta T, Iwanari H, Sakihama T, Kodama T, Hamakubo T, Shinkai Y: Histone methyltransferases G9a and GLP form heteromeric complexes and are both crucial for methylation of euchromatin at H3-K9. Genes Dev 2005, 19:815-826.

71. Metivier R, Penot G, Hubner MR, Reid G, Brand H, Kos M, Gannon F: Estrogen receptor-alpha directs ordered, cyclical, and combinatorial recruitment of cofactors on a natural target promoter. Cell 2003, 115:751-763.

72. Morin R, Bainbridge M, Fejes A, Hirst M, Krzywinski M, Pugh T, McDonald H, Varhol R, Jones S, Marra M: Profiling the HeLa S3 transcriptome using randomly primed cDNA and massively parallel short-read sequencing Biotechniques 2008, 45:81-94.

73. Li H, Ruan J, Durbin R: Mapping short DNA sequencing reads and calling variants using mapping quality scores. Genome Res 2008, 18:1851-1858.

74. Flicek P, Aken BL, Ballester B, Beal K, Bragin E, Brent S, Chen Y, Clapham P, Coates G, Fairley S, Fitzgerald S, Fernandez-Banet J, Gordon L, Gräf S, Haider S, Hammond M, Howe K, Jenkinson A, Johnson N, Kähäri A, Keefe D, Keenan S, Kinsella R, Kokocinski F, Koscielny G, Kulesha E, Lawson D, Longden I, Massingham T, McLaren W, et al: Ensembl's 10th year. Nucleic Acids Res 2010, 38:D557-D562.

75. Pruitt KD, Maglott DR: RefSeq and LocusLink: NCBI gene-centered resources. Nucleic Acids Res 2001, 29:137-140.

76. Rhead B, Karolchik D, Kuhn RM, Hinrichs AS, Zweig AS, Fujita PA, Diekhans M, Smith KE, Rosenbloom KR, Raney BJ, Pohl A, Pheasant M, Meyer LR, Learned K, Hsu F, Hillman-Jackson J, Harte RA, Giardine B, Dreszer TR, Clawson H, Barber GP, Haussler D, Kent WJ: The UCSC Genome Browser database: update 2010. Nucleic Acids Res 2010, 38:D613-D619.

77. Mortazavi A, Williams BA, McCue K, Schaeffer L, Wold B: Mapping and quantifying mammalian transcriptomes by RNA-Seq. Nat Methods 2008, 5:621-628.

78. Pepke S, Wold B, Mortazavi A: Computation for ChIP-seq and RNA-seq studies. Nat Methods 2009, 6:S22-S32.

79. Li H, Durbin R: Fast and accurate short read alignment with BurrowsWheeler transform. Bioinformatics 2009, 25:1754-1760.

80. Fejes AP, Robertson G, Bilenky M, Varhol R, Bainbridge M, Jones SJ: FindPeaks 3.1: a tool for identifying areas of enrichment from massively parallel shortread sequencing technology. Bioinformatics 2008, 24:1729-1730.

81. Kent WJ, Zweig AS, Barber G, Hinrichs AS, Karolchik D: BigWig and BigBed: enabling browsing of large distributed datasets. Bioinformatics 2010, 26:2204-2207

82. Dignam JD, Lebovitz RM, Roeder RG: Accurate transcription initiation by RNA polymerase II in a soluble extract from isolated mammalian nuclei. Nucleic Acids Res 1983, 11:1475-89.

83. Edgar R, Domrachev M, Lash AE: Gene Expression Omnibus: NCBI gene expression and hybridization array data repository. Nucleic Acids Res 2002, 30:207-10.

\section{doi:10.1186/1756-8935-6-15}

Cite this article as: Maksakova et al:: Distinct roles of KAP1, HP1 and G9a/GLP in silencing of the two-cell-specific retrotransposon MERVL in mouse ES cells. Epigenetics \& Chromatin 2013 6:15. 